\title{
Stereodivergent Syntheses of Anisomycin Derivatives from D-Tyrosine
}

\author{
Jin Hyo Kim, Marcus J. Curtis-Long ${ }^{\dagger}$, Woo Duck Seo, Young Bae Ryu, Min Suk Yang, \\ and Ki Hun Park ${ }^{\ddagger} *$
}

Department of Agricultural Chemistry, Division of Applied Life Science (BK21 programs), Gyeongsang National University, Jinju, 660-701, South Korea.

† Trout Beck, Wansford, Driffield, East Yorkshire YO25 8NX, UK.

${ }^{\ddagger}$ Institute of Agriculture \& Life sciences, Gyeongsang National University, Jinju, 660-701, South Korea

\section{Supporting Information}

1. General Procedures

2. Synthesis and Spectroscopic Data

(Compound 5, 6, 16, 17 and 19)

3. NMR and Mass Spectrum

(Compound 2 12, 14 17, 19)

Corresponding Author: Professor Ki Hun Park; Tel. +82-55-751-5472, Fax. +82-757-0178, e-mail : khpark@gsnu.ac.kr 


\section{General Procedures}

All non-aqueous reaction was carried out under an inert nitrogen atmosphere. THF was distilled from $\mathrm{Na}$ /benzophenone; triethylamine $\left(\mathrm{Et}_{3} \mathrm{~N}\right)$, 2,2-dimethoxypropane, $\mathrm{DMF}$, and methylene chloride were distilled from $\mathrm{CaH}_{2}$. All reactions were monitored by TLC which using commercially available glassbacked plates. Column chromatography was carried out using 230-400 mesh silica gel. Final solution before evaporation was washed with brine and dried over anhydrous $\mathrm{Na}_{2} \mathrm{SO}_{4}$. Infrared spectra were measured with $\mathrm{KBr}$. Proton and carbon magnetic resonance spectra were measured downfield relative to tetramethylsilane in $\mathrm{CDCl}_{3}$ unless otherwise noted (values in $\mathrm{ppm}$ ). Melting points are uncorrected. $[\alpha]_{\mathrm{D}}$-values are given in units of $10^{-1} \mathrm{deg}_{\mathrm{cm}^{2} \mathrm{~g}^{-1}}$.

(S)- or (R)-Binaphtol-modified lithium aluminium hydride reagents [(S)- or (R)-BINAL $]^{25}$

BINAL was prepared by treating $\mathrm{LiAlH}_{4}$ in THF $(1.0 \mathrm{M}$ solution) with equimolar amounts of methanol (1.0 M solution in THF) and opically pure (S)-(-)-2,2'-dihydroxy-1,1'-binaphthyl (0.3 $\mathrm{M}$ in THF solution) for $1 \mathrm{~h}$ at room temperature.

\section{(2R)-3-p-Methoxyphenyl-2-(N-9-phenylfluoren-9-yl) amino-1-propanal (6)}

This was prepared as described ${ }^{23} \cdot[\alpha]_{\mathrm{D}}+1.6\left(c\right.$ 1.0, $\left.\mathrm{CHCl}_{3}\right)$; IR $(\mathrm{KBr}): 3068,2940,1726 \mathrm{~cm}^{-1} ;{ }^{1} \mathrm{H}$ NMR $\left(500 \mathrm{MHz} ; \mathrm{CDCl}_{3}\right) \delta 2.57(2 \mathrm{H}, \mathrm{m}), 2.67(1 \mathrm{H}, \mathrm{m}), 3.78(3 \mathrm{H}, \mathrm{s}), 7.62 \sim 6.67(17 \mathrm{H}, \mathrm{m}) ;{ }^{13} \mathrm{C} \mathrm{NMR}$ $\left(125 \mathrm{MHz} ; \mathrm{CDCl}_{3}\right) \delta 34.8,53.3,61.0,70.8,112.1,117.8,118.0,122.9,123.7,124.1,125.3,125.8$, 125.9, 126.3, 126.4, 126.9, 128.5, 138.6, 138.7, 142.3, 146.7, 146.8, 156.6, 200.9 (Found; C, 83.11; H, 6.12; $\mathrm{N}, 3.29 . \mathrm{C}_{29} \mathrm{H}_{25} \mathrm{NO}_{2}$ requires $\left.\mathrm{C}, 83.03 ; \mathrm{H}, 6.01 ; \mathrm{N}, 3.34 \%\right)$.

\section{(3R, 4R)-4-(N-9-phenylfluoren-9-yl)amino-3-acetoxy-5-(p-methoxyphenyl)-1-pentene (16)}

Compound 16 (1.58 g, $96 \%)$ was prepared from compound $7 \mathbf{b}(1.50 \mathrm{~g}, 3.35 \mathrm{mmol})$ according to the same procedures for compound 9 as a solid. mp. 52; $[\alpha]_{\mathrm{D}}-37.4$ (c 5.0, $\left.\mathrm{CHCl}_{3}\right)$; IR (KBr): 3063, 2934, $1737 \mathrm{~cm}^{-1} ;{ }^{1} \mathrm{H}$ NMR (500 MHz; $\left.\mathrm{CDCl}_{3}\right) \delta 1.94(3 \mathrm{H}, \mathrm{s}), 2.21(2 \mathrm{H}, \mathrm{m}), 2.48(2 \mathrm{H}, \mathrm{m}), 3.74(3 \mathrm{H}, \mathrm{s}), 4.82$ 
$(1 \mathrm{H}, \mathrm{m}), 5.09(1 \mathrm{H}, \mathrm{d}, J=17.3 \mathrm{~Hz}), 5.20(1 \mathrm{H}, \mathrm{d}, J=10.7 \mathrm{~Hz}), 5.90(1 \mathrm{H}, \mathrm{m}), 7.71 \sim 6.58(17 \mathrm{H}, \mathrm{m}) ;{ }^{13} \mathrm{C}$ NMR (125 MHz; $\left.\mathrm{CDCl}_{3}\right) \delta 20.9,37.6,55.2,57.4,72.7,74.9,98.5,113.7,116.6,119.7,120.1,125.3$, $126.0,126.1,127.1,127.6,127.7,128.1,128.2,128.4,130.2,131.0,134.3,140.2,140.8,145.6,149.3$, 149.9, 158.0, 169.5 (Found; C, 81.08; H, 6.49; N, 2.97. $\mathrm{C}_{33} \mathrm{H}_{31} \mathrm{NO}_{3}$ requires $\mathrm{C}, 80.95 ; \mathrm{H}, 6.38$; N, $2.86 \%)$.

\section{(2R, 3S, 4R)-4-(N-9-phenylfluoren-9-yl)-3-acetoxy-4-iodo-2-(p-methoxybenzyl) pyrrolidine (17)}

Compound 17 (1.8 g, $90 \%$ ) was prepared from compound 16 (1.58 g, $3.22 \mathrm{mmol})$ according to the same procedures for compound 10. mp. $73{ }^{\circ} \mathrm{C} ;[\alpha]_{\mathrm{D}}+55.0$ (c 3.0, $\left.\mathrm{CHCl}_{3}\right)$; IR (KBr): 3060, 2952, 1747 $\mathrm{cm}^{-1} ;{ }^{1} \mathrm{H}$ NMR $\left(500 \mathrm{MHz} ; \mathrm{CDCl}_{3}\right) \delta 2.08(3 \mathrm{H}, \mathrm{s}), 2.19(1 \mathrm{H}, \mathrm{d}, J=14.0 \mathrm{~Hz}), 2.64(1 \mathrm{H}, \mathrm{m}), 2.86(1 \mathrm{H}, \mathrm{dd}$, $J=14.0,10.9 \mathrm{~Hz}), 3.17(1 \mathrm{H}, \mathrm{dd}, J=11.2,9.2 \mathrm{~Hz}), 3.43(1 \mathrm{H}, \mathrm{m}), 3.55(1 \mathrm{H}, \mathrm{m}), 3.69(3 \mathrm{H}, \mathrm{s}), 5.17(1 \mathrm{H}, \mathrm{t}$, $J=6.9 \mathrm{~Hz}), 6.57(2 \mathrm{H}, \mathrm{d}, J=8.6 \mathrm{~Hz}), 6.64(2 \mathrm{H}, \mathrm{d}, J=8.6 \mathrm{~Hz}), 7.74 \sim 7.24(17 \mathrm{H}, \mathrm{m}) ;{ }^{13} \mathrm{C} \mathrm{NMR}(125$ $\left.\mathrm{MHz} ; \mathrm{CDCl}_{3}\right) \delta 3.9,21.0,35.8,55.1,62.1,62.6,65.8,75.7,113.5,120.1,120.2,125.4,126.6,127.4$, $127.5,127.9,128.0,128.1,128.2,128.5,128.9,129.0,129.1,129.3,140.4,141.5,145.8,146.1,150.5$, 157.8, 169.7 (Found; C, 64.45; H, 5.04; N, 2.36. $\mathrm{C}_{33} \mathrm{H}_{30} \mathrm{NO}_{3} \mathrm{I}$ requires $\mathrm{C}, 64.40 ; \mathrm{H}, 4.91 ; \mathrm{N}, 2.28 \%$ ).

\section{(2R, 3S, 4R)-(N-9-Phenylfluoren-9-yl)-deacetyl anisomycin (19)}

Compound 19 (0.47 g, $70 \%)$ was prepared from compound $17(0.9 \mathrm{~g}, 1.46 \mathrm{mmol})$ according to the same procedures for compound 14 as a solid. mp. $86{ }^{\circ} \mathrm{C} ;[\alpha]_{\mathrm{D}}+147.3$ (c 1.0, $\left.\mathrm{CHCl}_{3}\right)$; IR (KBr): 3313 , 3023, $2970 \mathrm{~cm}^{-1} ;{ }^{1} \mathrm{H}$ NMR (300 MHz; $\left.\mathrm{CDCl}_{3}\right) \delta 1.28(1 \mathrm{H}, \mathrm{OH}), 2.21(1 \mathrm{H}, \mathrm{OH}), 2.73(2 \mathrm{H}, \mathrm{m}), 3.27(2 \mathrm{H}$, m), $3.54(1 \mathrm{H}, \mathrm{m}), 3.74(3 \mathrm{H}, \mathrm{s}), 3.97(1 \mathrm{H}, \mathrm{m}), 7.79 \sim 6.72(17 \mathrm{H}, \mathrm{m}) ;{ }^{13} \mathrm{C} \mathrm{NMR}\left(75 \mathrm{MHz} ; \mathrm{CDCl}_{3}\right) \delta 31.6$, $35.9,55.2,56.2,70.5,72.8,77.2,113.6,119.7,120.0,125.6,127.0,127.1,127.7,128.0,128.4,130.0$, 139.4, 141.5, 148.1, 157.7 (Found; C, 80.43; H, 6.43; N, 3.08. $\mathrm{C}_{31} \mathrm{H}_{29} \mathrm{NO}_{3}$ requires $\mathrm{C}, 80.32 ; \mathrm{H}, 6.31$; N, $3.02 \%)$.

\section{$(2 R, 3 S, 4 R)$-Deacetyl anisomycin (5)}


Compound $5(0.24 \mathrm{~g}, 92 \%)$ was prepared from compound $19(0.47 \mathrm{~g}, 1.01 \mathrm{mmol})$ according to the same procedures for compound 4. $[\alpha]_{\mathrm{D}}+6.0$ (c 0.4, MeOH); IR $(\mathrm{KBr}): 3304,3023,2970 \mathrm{~cm}^{-1} ;{ }^{1} \mathrm{H}$ NMR (300 MHz; $\left.\mathrm{CDCl}_{3}\right) \delta 2.92(1 \mathrm{H}, \mathrm{dd}, J=13.9,8.0 \mathrm{~Hz}), 3.16(2 \mathrm{H}, \mathrm{m}), 3.37(1 \mathrm{H}, \mathrm{m}), 3.64(1 \mathrm{H}, \mathrm{m})$, $3.78(3 \mathrm{H}, \mathrm{s}), 3.99(1 \mathrm{H}, \mathrm{m}), 4.38(1 \mathrm{H}, \mathrm{m}), 6.90(2 \mathrm{H}, \mathrm{d}, J=8.6 \mathrm{~Hz}), 7.25(2 \mathrm{H}, \mathrm{d}, J=8.6 \mathrm{~Hz}) ;{ }^{13} \mathrm{C} \mathrm{NMR}$ $\left(75 \mathrm{MHz} ; \mathrm{CDCl}_{3}\right) \delta 31.7,47.8,54.2,63.7,70.4,70.7,113.9,128.3,129.7,158.9 ; \mathrm{MS}-\mathrm{EI} \mathrm{m} / \mathrm{z} 223\left(\mathrm{M}^{+}\right)$, 144, 121, 102; HRMS calcd for $\mathrm{C}_{12} \mathrm{H}_{17} \mathrm{NO}_{3} 223.1208$, found 223.1213 . 


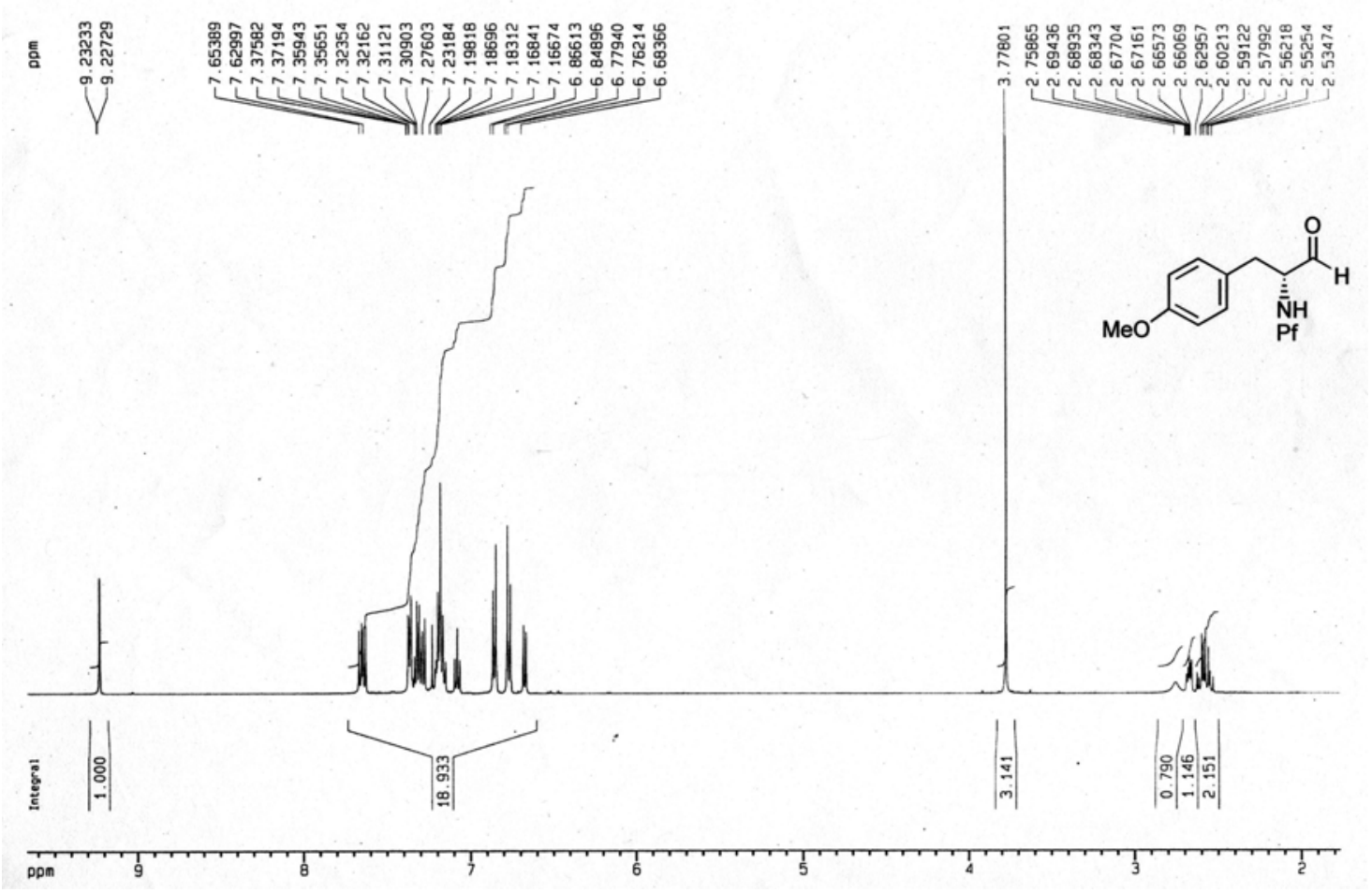

Spectra 1. ${ }^{1} \mathrm{H}$ NMR of (2R)-3-p-Methoxyphenyl-2-(N-9-phenylfluoren-9-yl) amino-1-propanal (6)

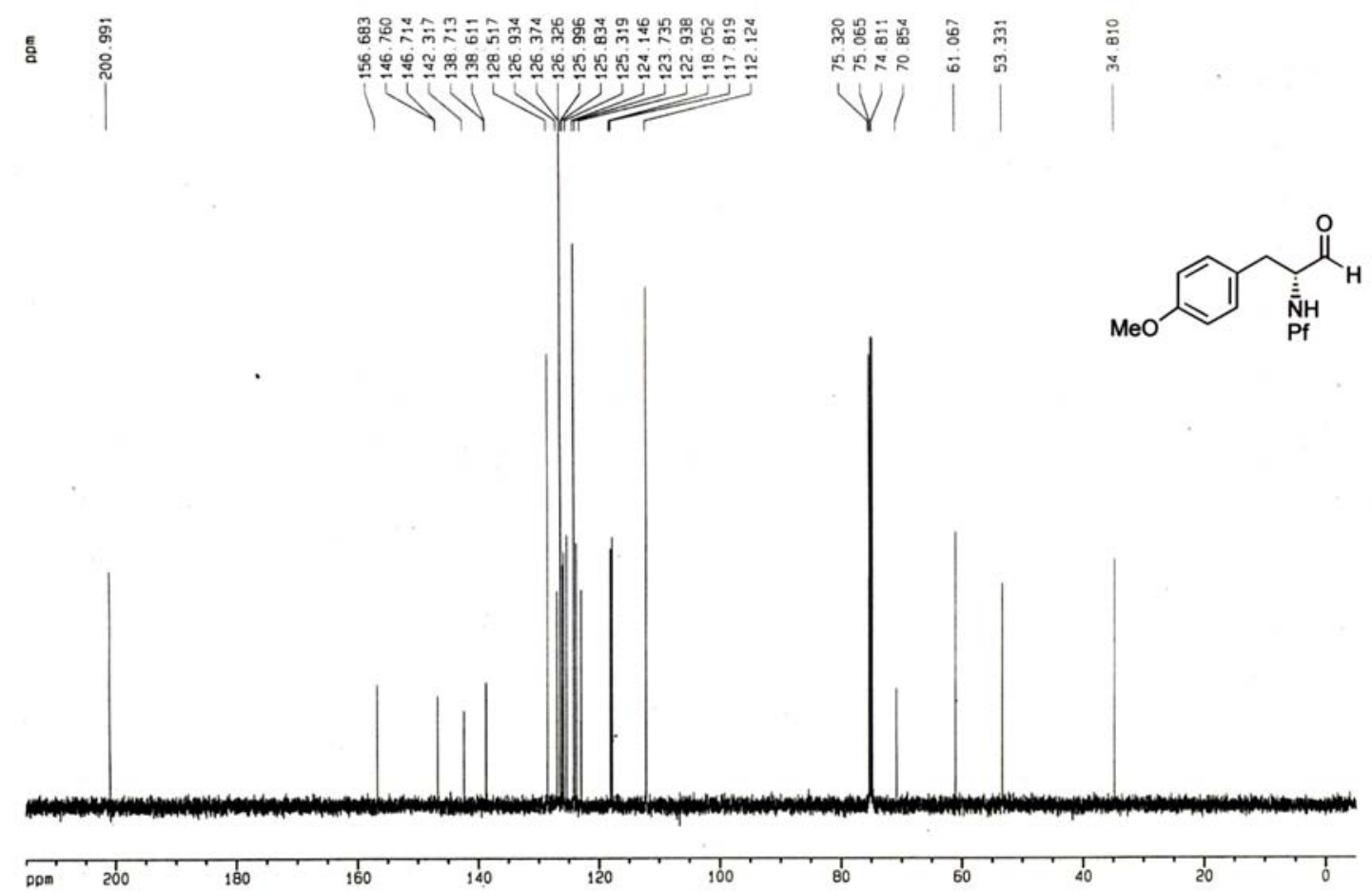

Spectra $2 .{ }^{13} \mathrm{C}$ NMR of (2R)-3-p-Methoxyphenyl-2-(N-9-phenylfluoren-9-yl) amino-1-propanal

(6) 


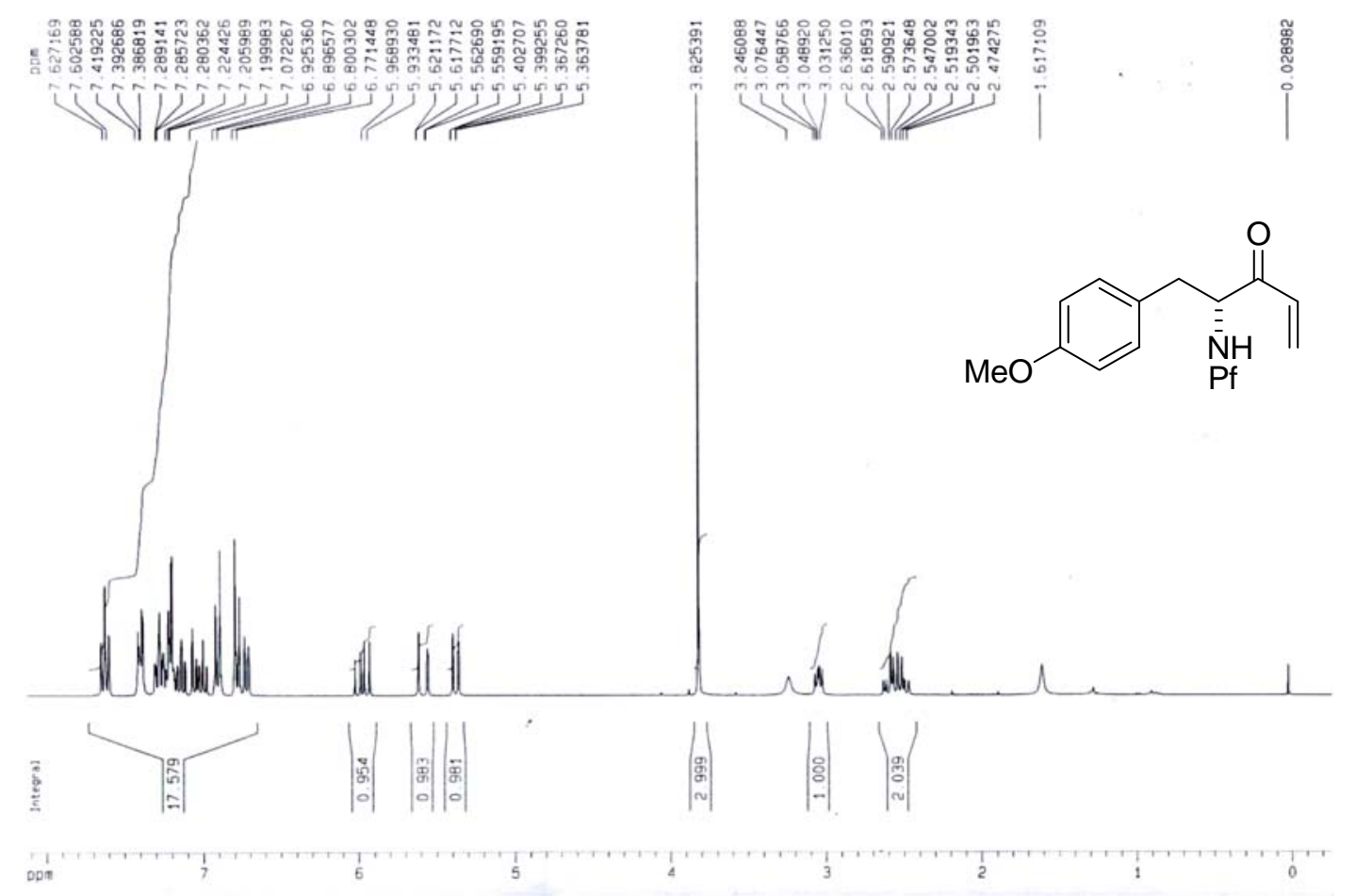

Spectra 3. ${ }^{1} \mathrm{H}$ NMR of (4R)-5-(p-Methoxyphenyl)-4-( $N$-9-phenylfluoren-9-yl) amino-pent-1-en-3-one (8)

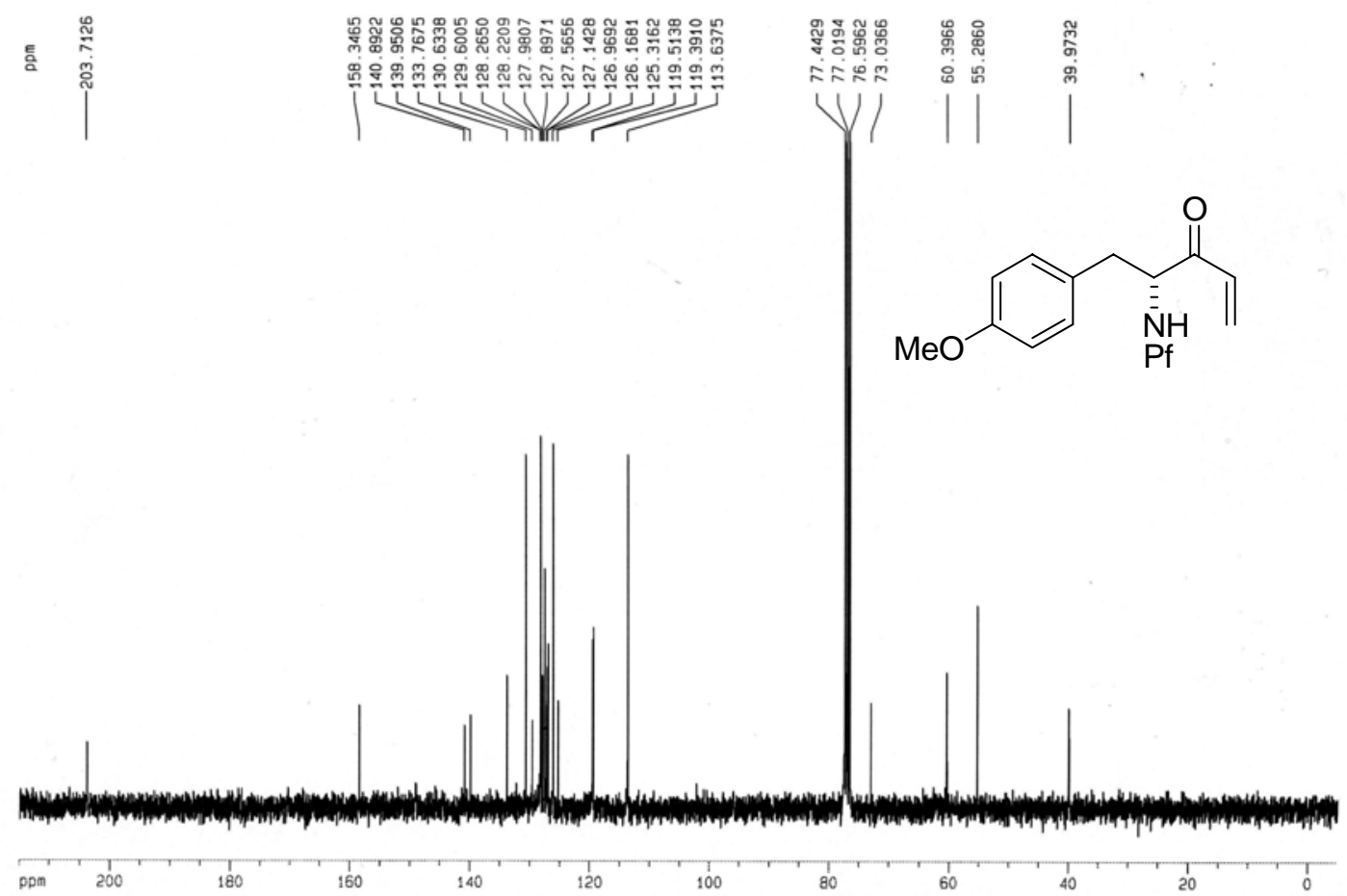

Spectra $4 .{ }^{13} \mathrm{C}$ NMR of (4R)-5-(p-Methoxyphenyl)-4-( $\boldsymbol{N}$-9-phenylfluoren-9-yl) amino-pent-1-en-3-one (8) 


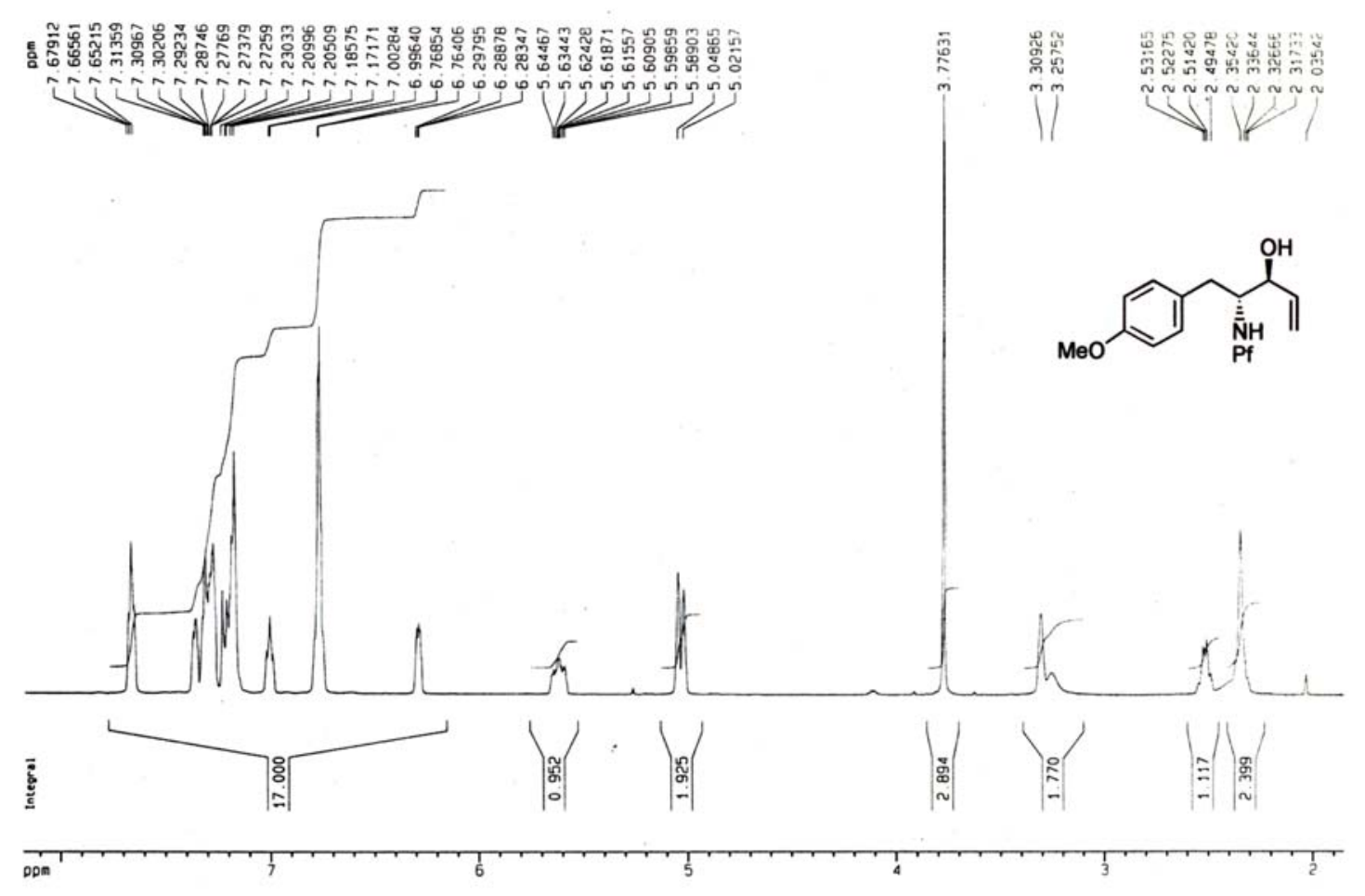

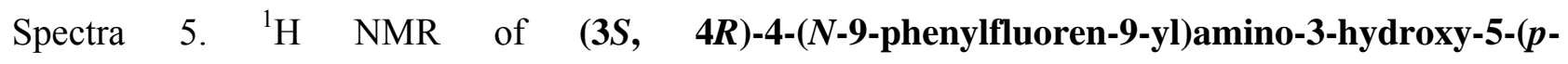
methoxyphenyl)-1-pentene (7a)

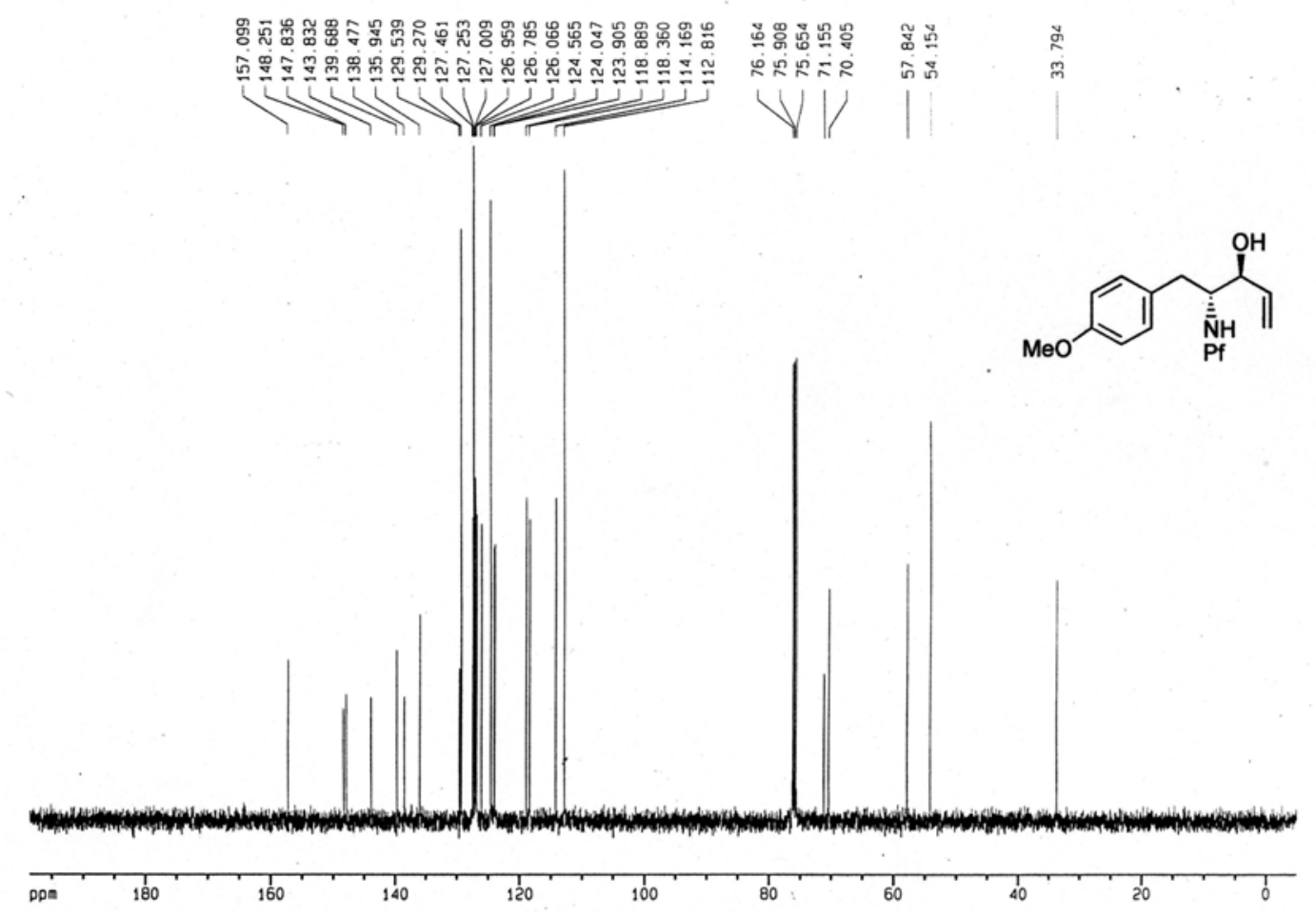

Spectra $\quad 6 . \quad{ }^{13} \mathrm{C} \quad \mathrm{NMR} \quad$ of $\quad(3 S, \quad 4 R)-4-(N-9-p h e n y l f l u o r e n-9-y l) a m i n o-3-h y d r o x y-5-(p-$ methoxyphenyl)-1-pentene (7a) 


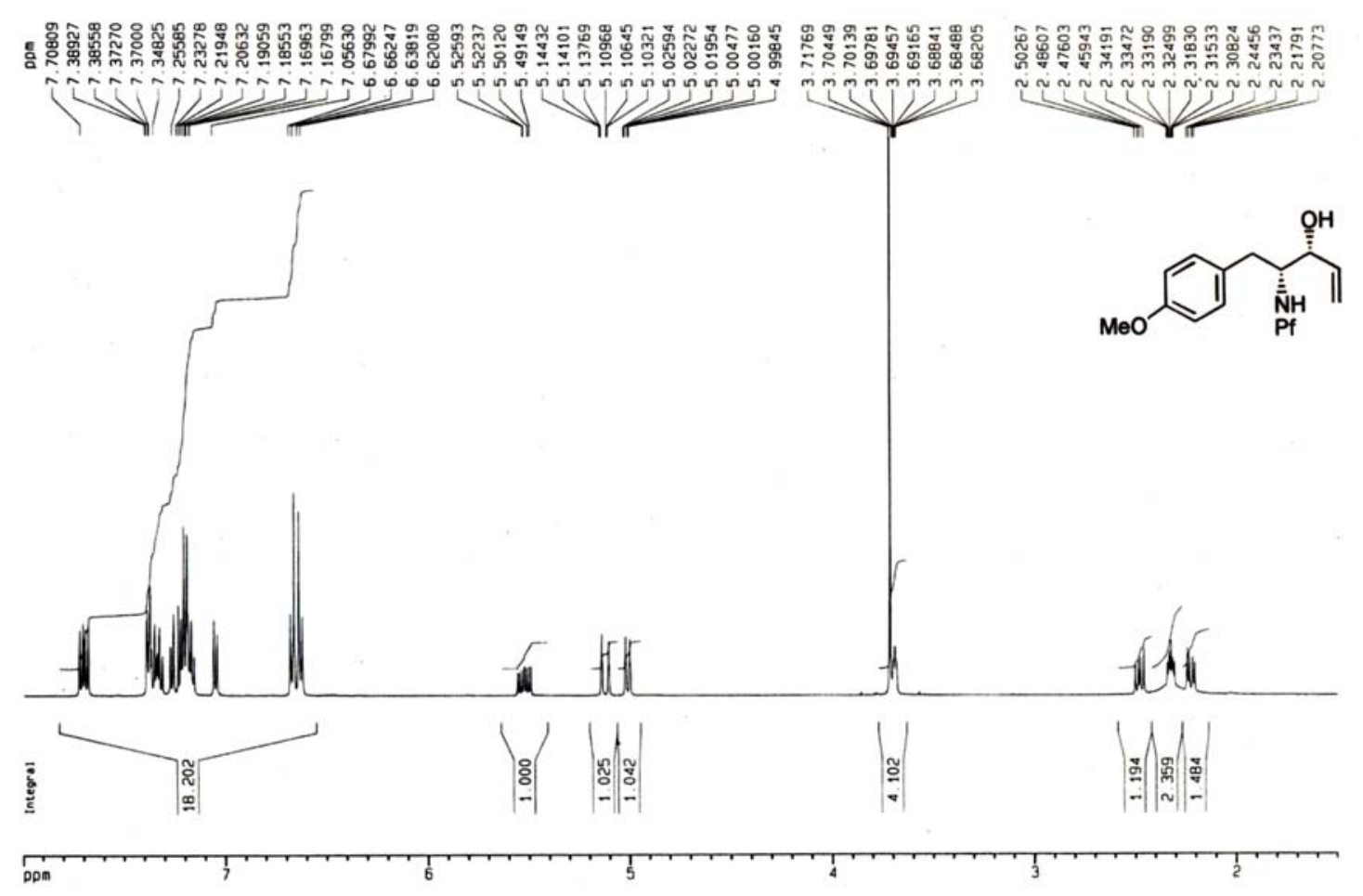

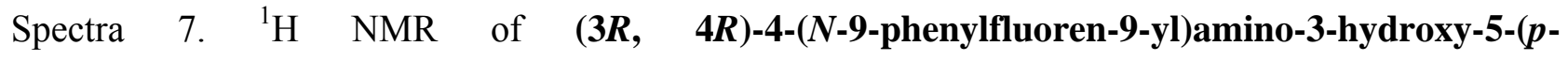
methoxyphenyl)-1-pentene (7b)

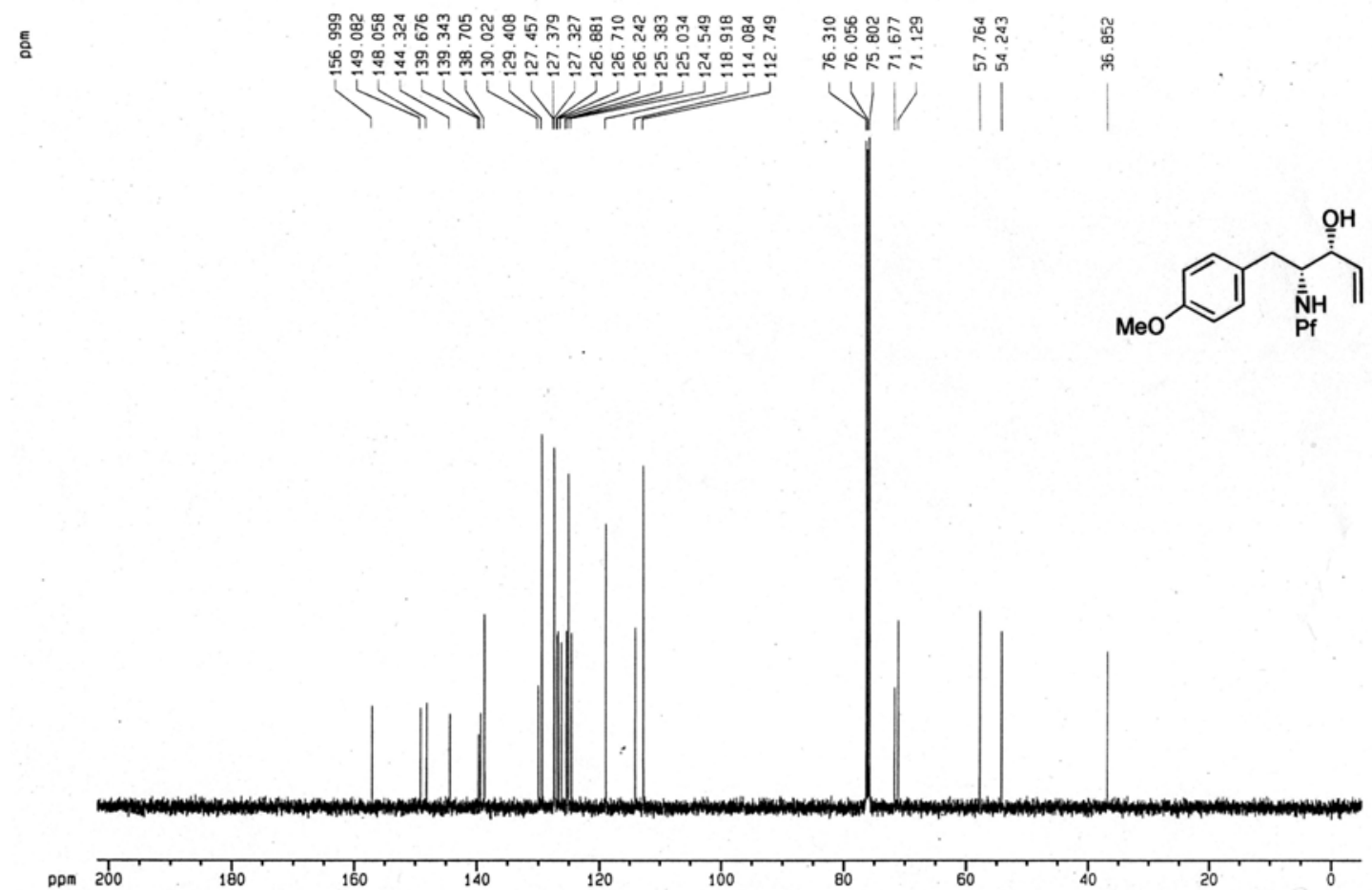

Spectra $\quad 8 . \quad{ }^{13} \mathrm{C} \quad \mathrm{NMR} \quad$ of $\quad(3 \boldsymbol{R}, \quad \mathbf{4 R})-\mathbf{4}-(\boldsymbol{N}-\mathbf{9}$-phenylfluoren-9-yl)amino-3-hydroxy-5-( $\boldsymbol{p}$ methoxyphenyl)-1-pentene (7b) 


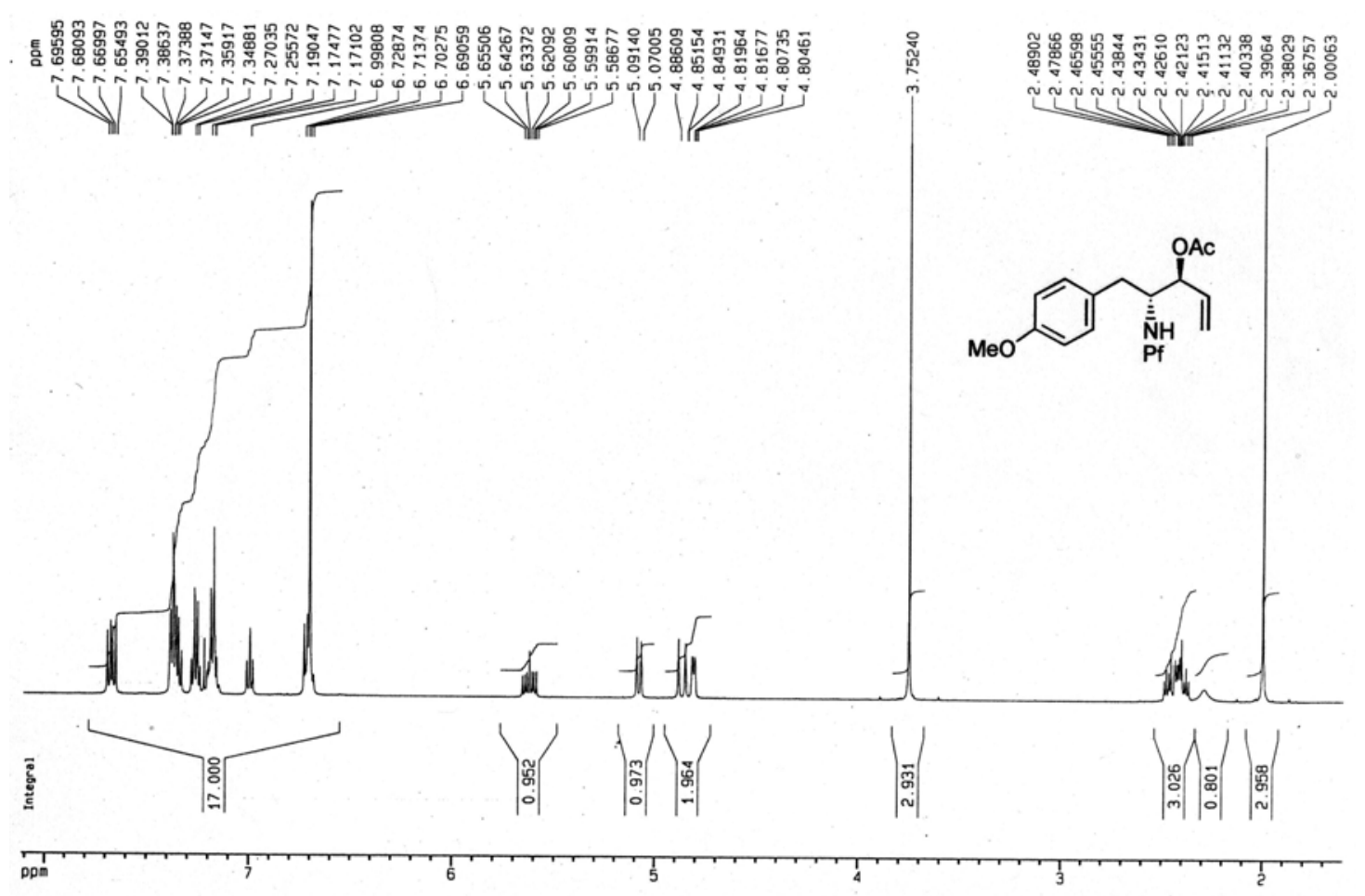

Spectra 9. ${ }^{1} \mathrm{H}$ NMR of (3S, 4R)-4-( $N$-9-phenylfluoren-9-yl)amino-3-acetoxy-5-(p-methoxyphenyl)1-pentene (9)

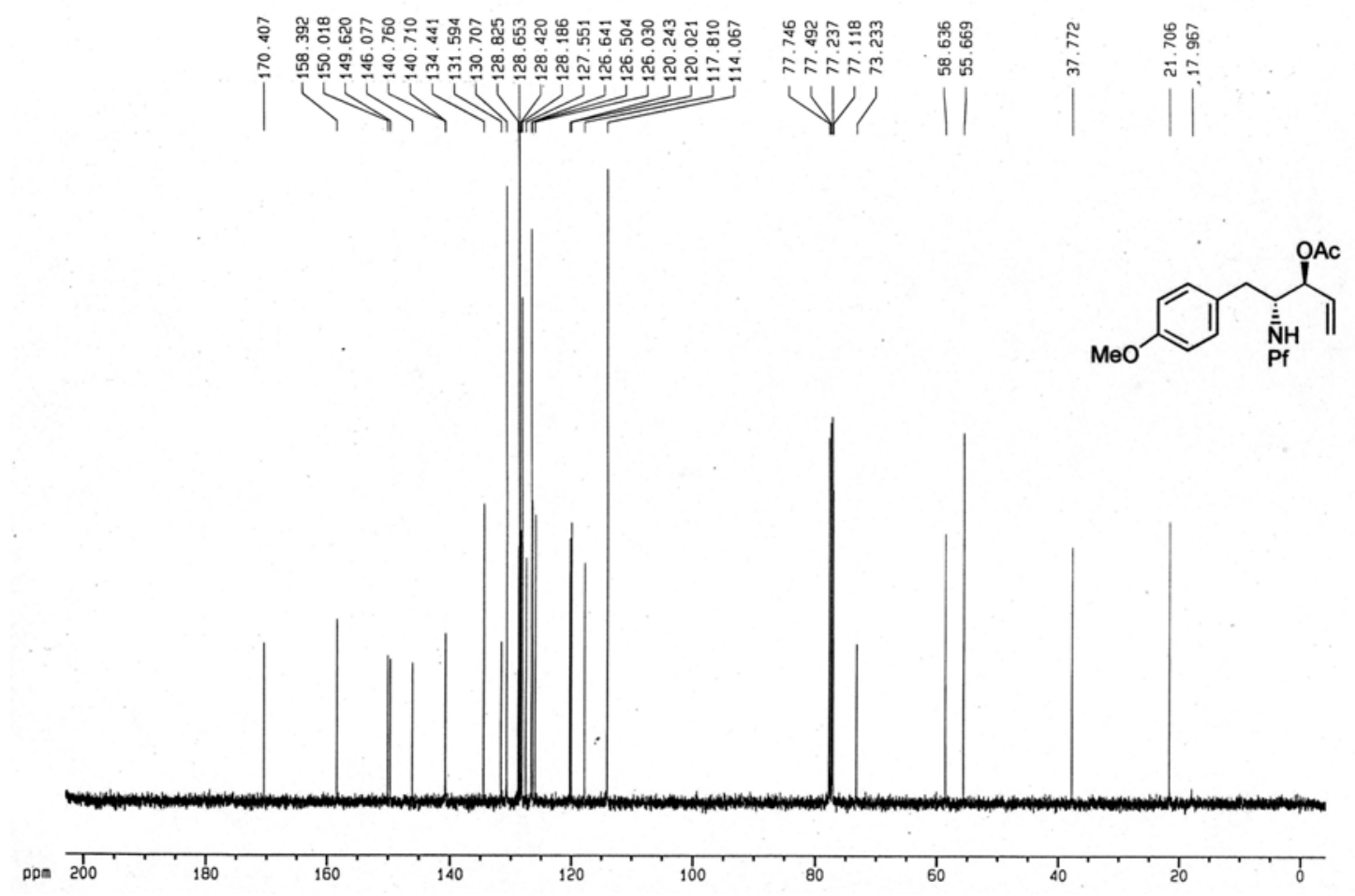

Spectra $\quad 10 . \quad{ }^{13} \mathrm{C} \quad$ NMR of $\quad(3 S, \quad 4 R)-4-(N-9-p h e n y l f l u o r e n-9-y l) a m i n o-3-a c e t o x y-5-(p-$ methoxyphenyl)-1-pentene (9) 


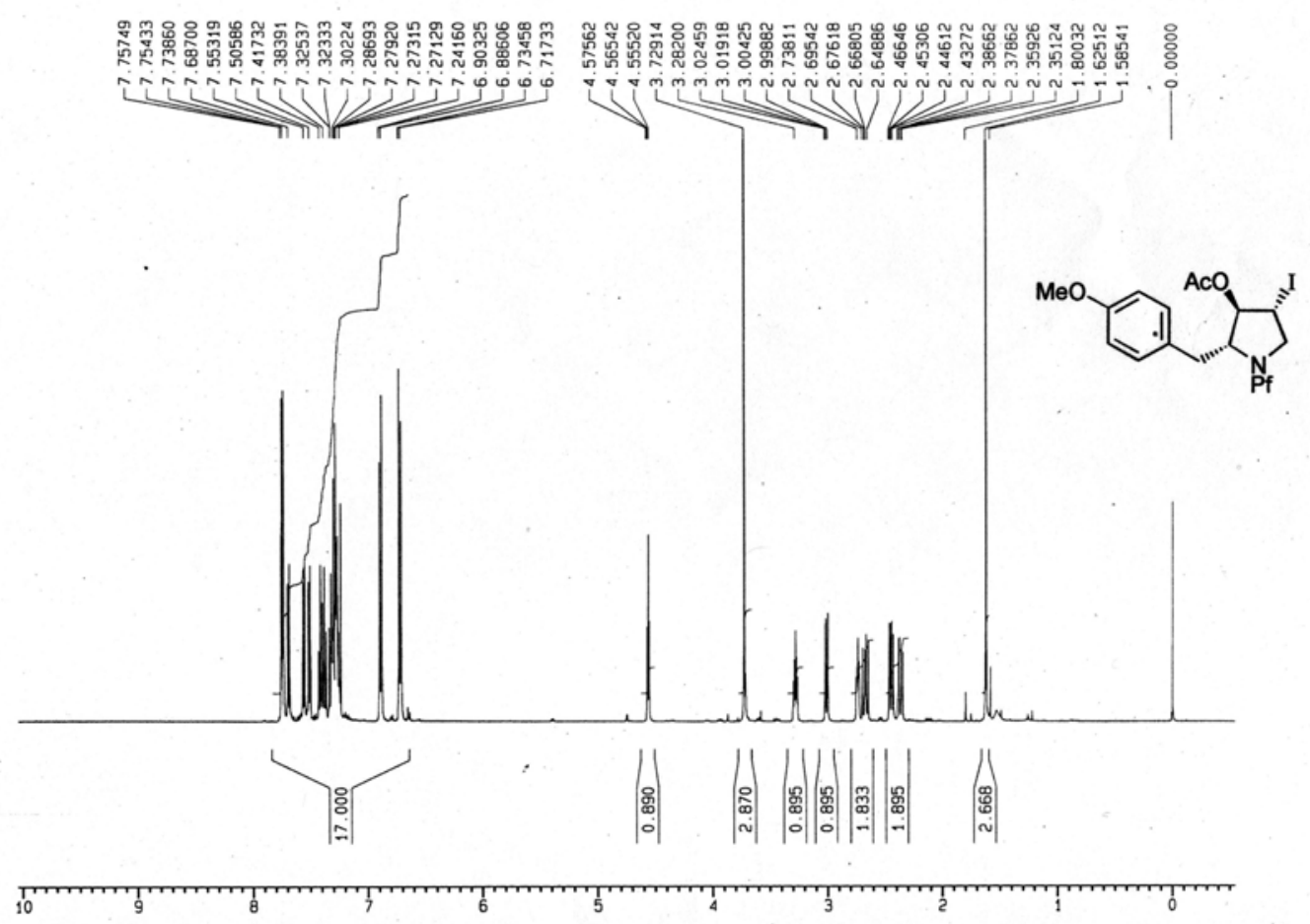

Spectra 11. ${ }^{1} \mathrm{H} \quad$ NMR of $\quad(2 \boldsymbol{R}, \quad 3 \boldsymbol{R}, \quad \mathbf{4})$-4-( $\boldsymbol{N - 9}$-phenylfluoren-9-yl)-3-acetoxy-4-iodo-2-(pmethoxybenzyl) pyrrolidine (10)

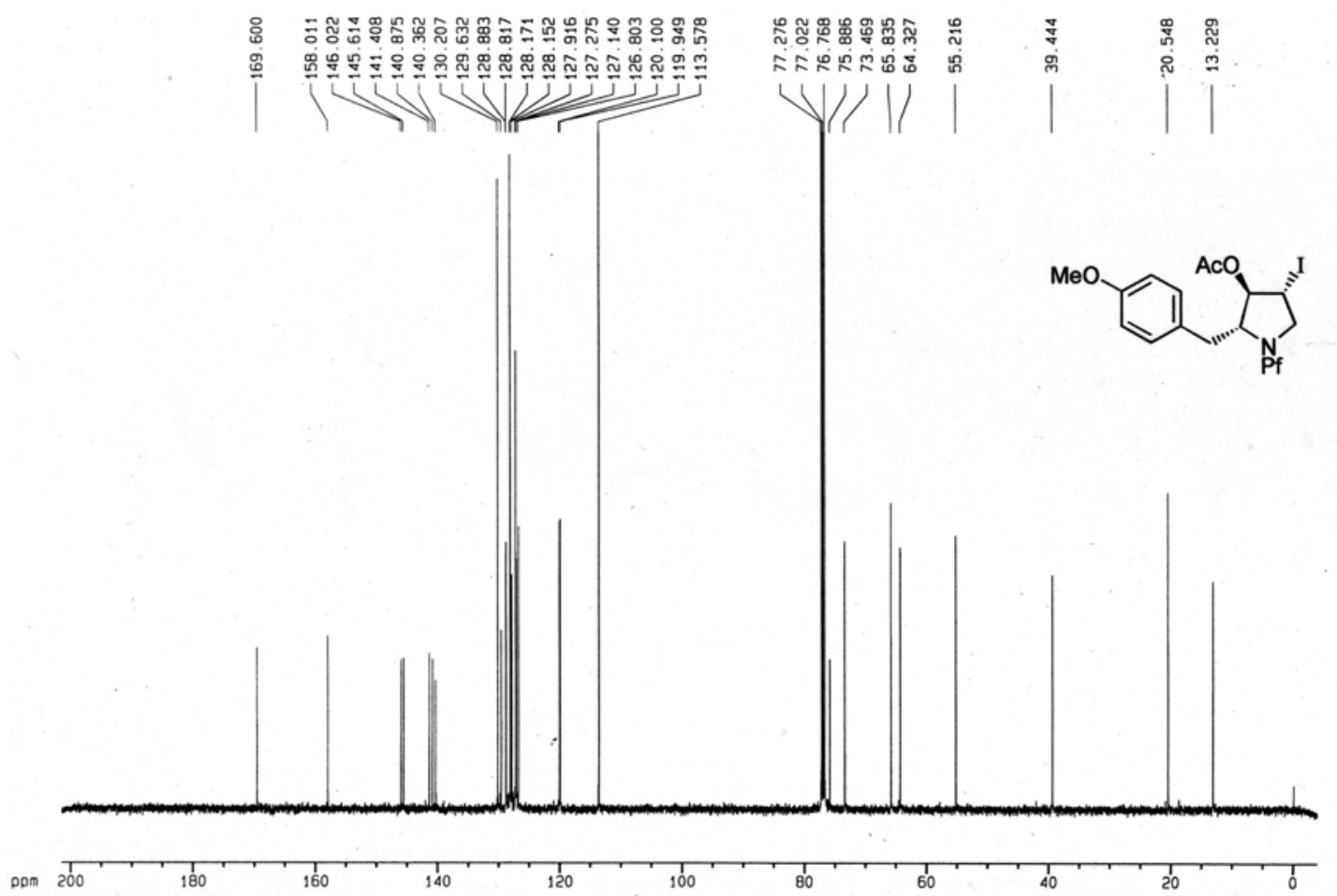

Spectra $12 .{ }^{13} \mathrm{C} \quad \mathrm{NMR}$ of $(\mathbf{2} \boldsymbol{R}, \quad 3 \boldsymbol{R}, \quad \mathbf{4} \boldsymbol{R})-\mathbf{4}-(\boldsymbol{N}-\mathbf{9}$-phenylfluoren-9-yl)-3-acetoxy-4-iodo-2-(pmethoxybenzyl) pyrrolidine (10) 


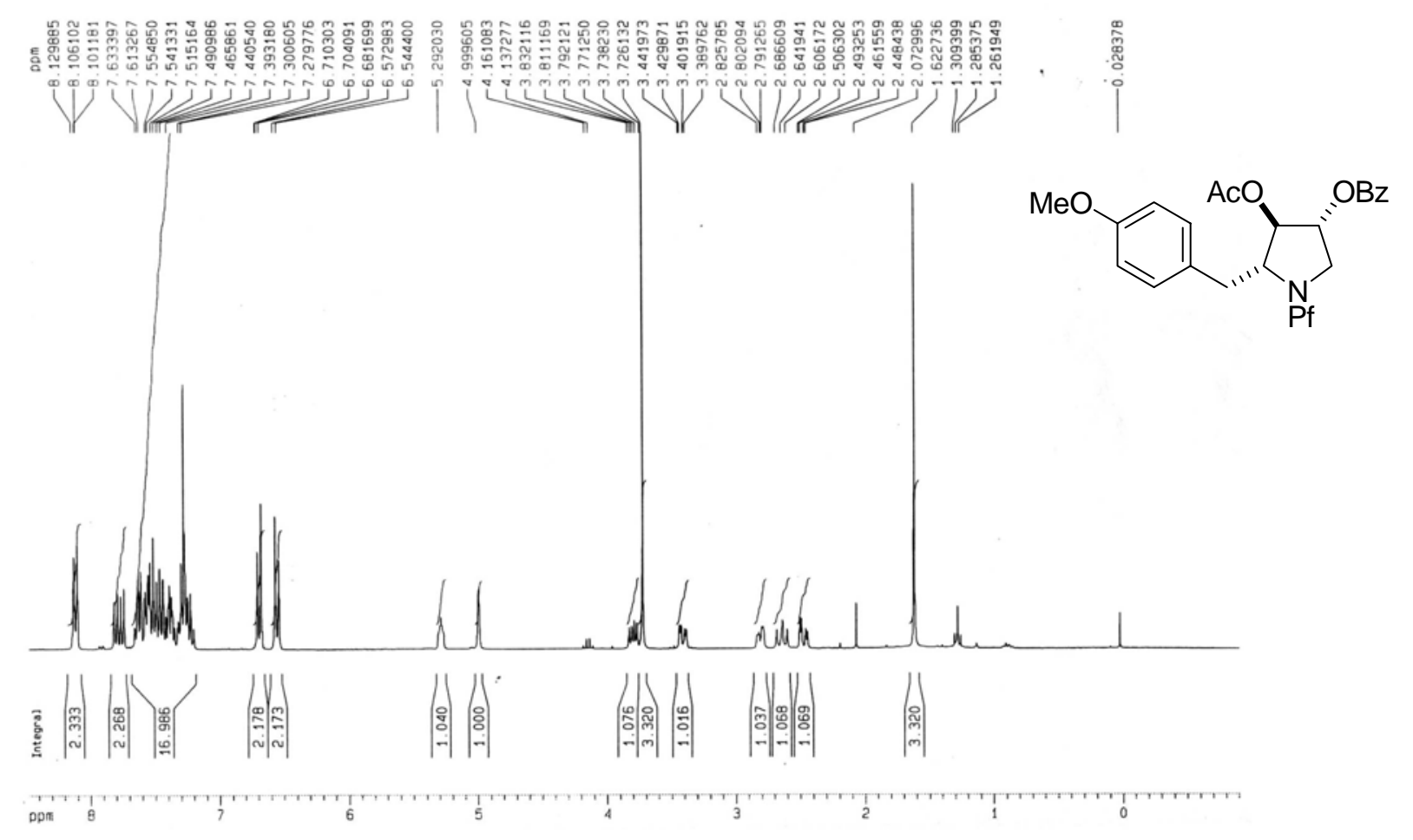

Spectra 13. ${ }^{1} \mathrm{H}$ NMR of (2R, 3R, 4R)-(N-9-phenylfluoren-9-yl)-4-O-benzoyl anisomycin (11)

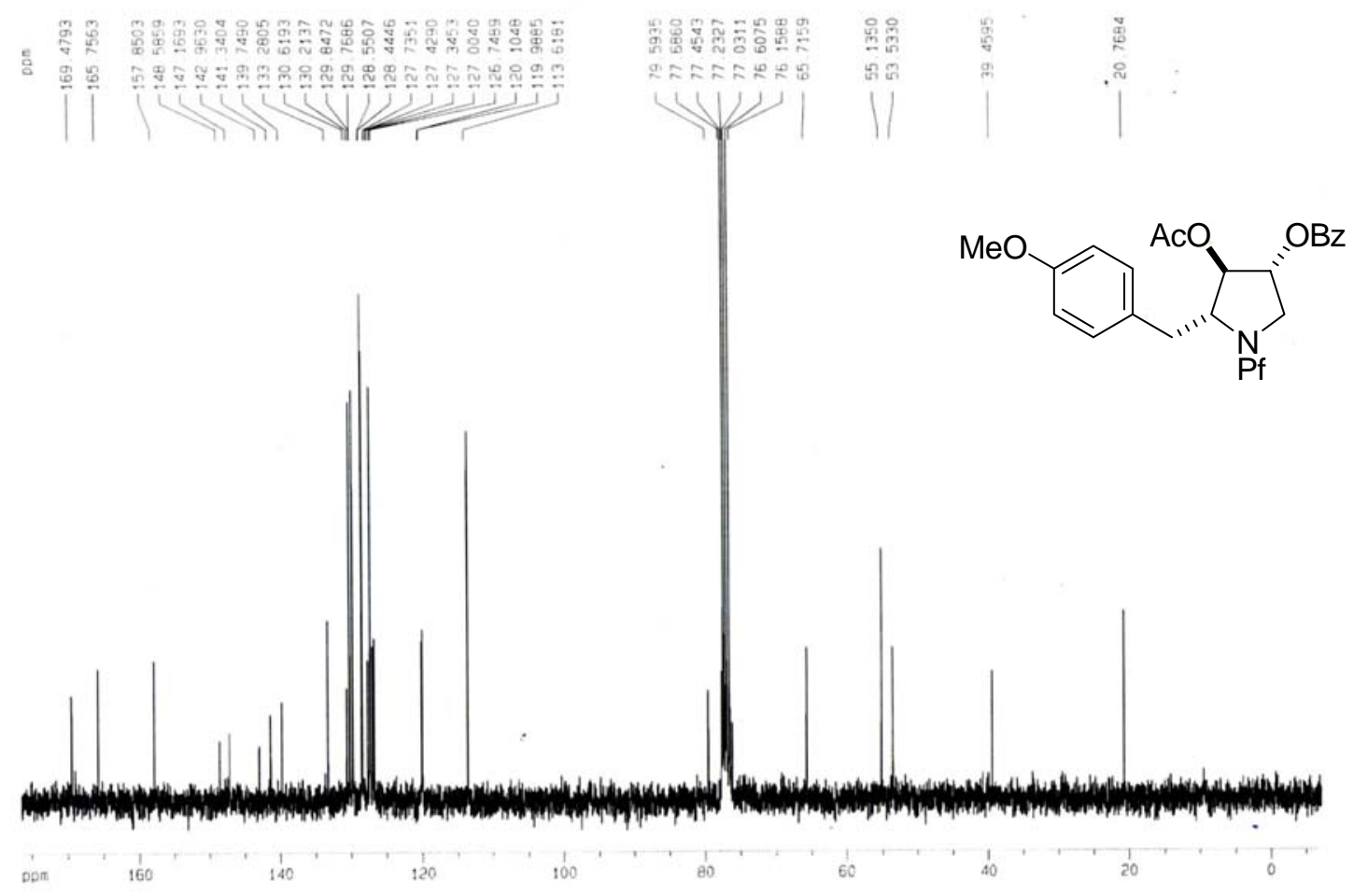

Spectra $14 .{ }^{13} \mathrm{C}$ NMR of $(2 \boldsymbol{R}, \mathbf{3 R}, \mathbf{4 R})-(\boldsymbol{N}-\mathbf{9}$-phenylfluoren-9-yl)-4-O-benzoyl anisomycin (11) 


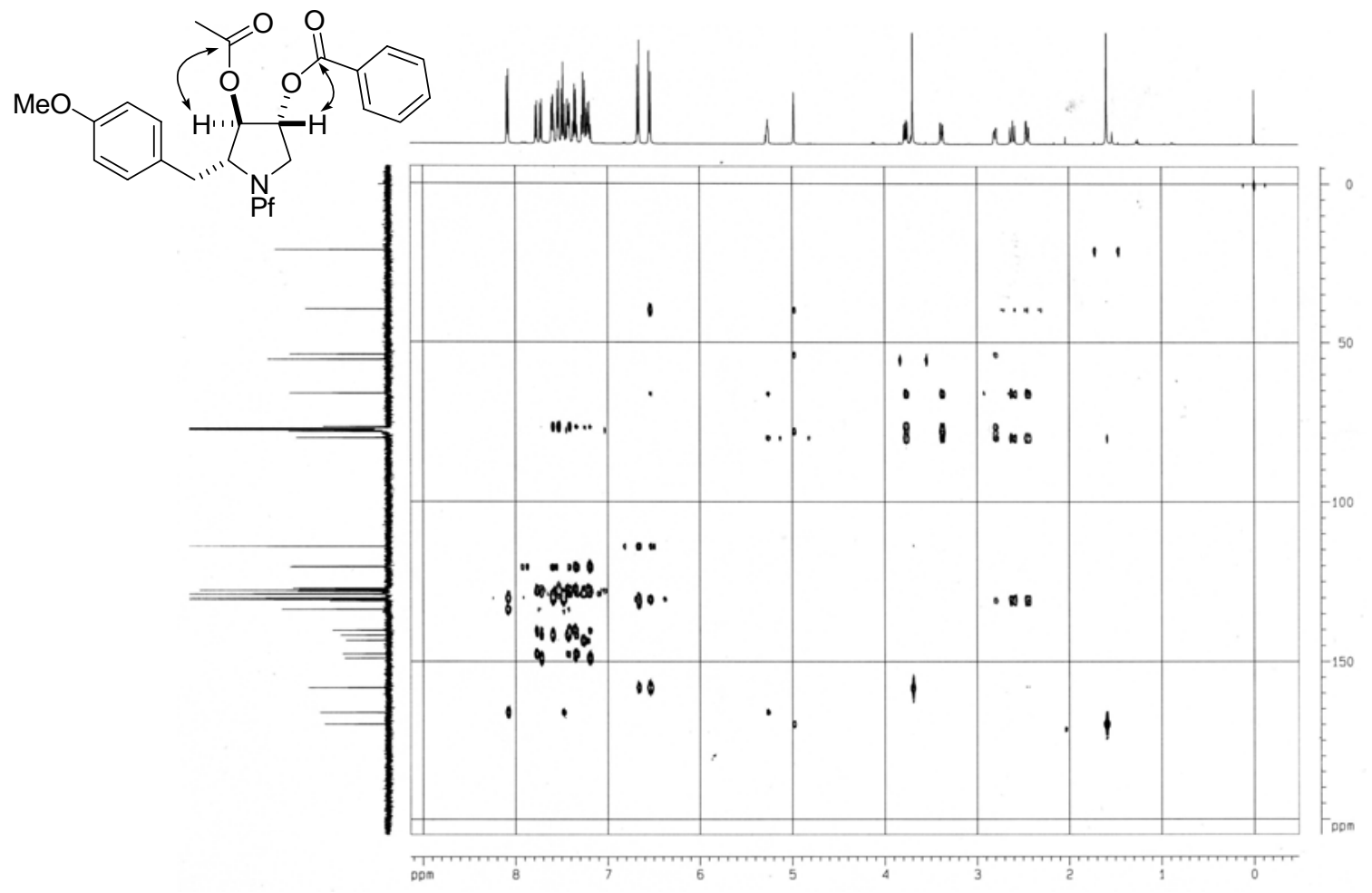

Spectra 15. HMBC of $(2 R, 3 R, 4 R)-(N-9$-phenylfluoren-9-yl)-4-O-benzoyl anisomycin (11) 


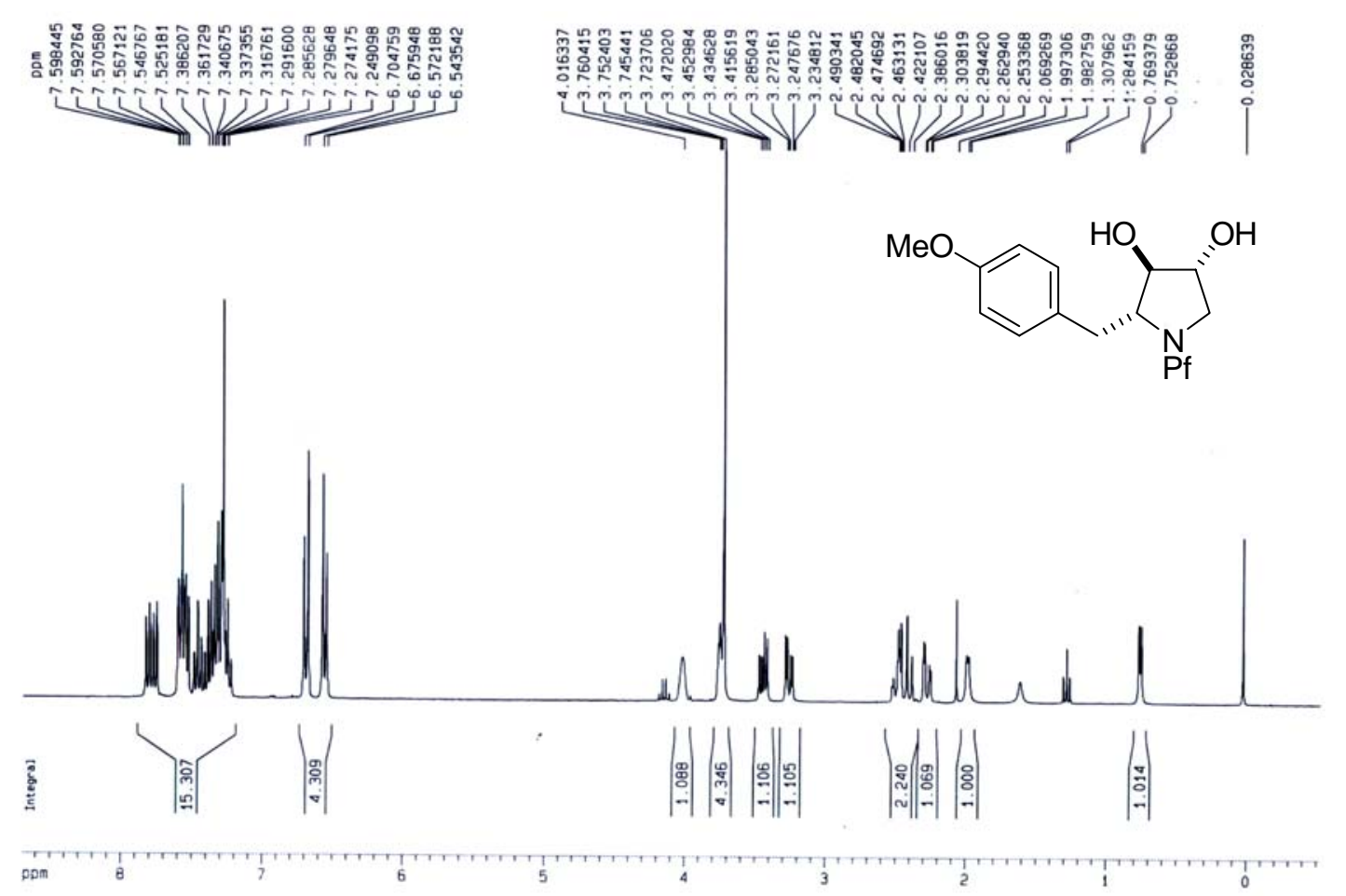

Spectra $16 .{ }^{1} \mathrm{H}$ NMR of (2R, 3R, 4R)-(N-9-phenylfluoren-9-yl)-deacetyl anisomycin (12)

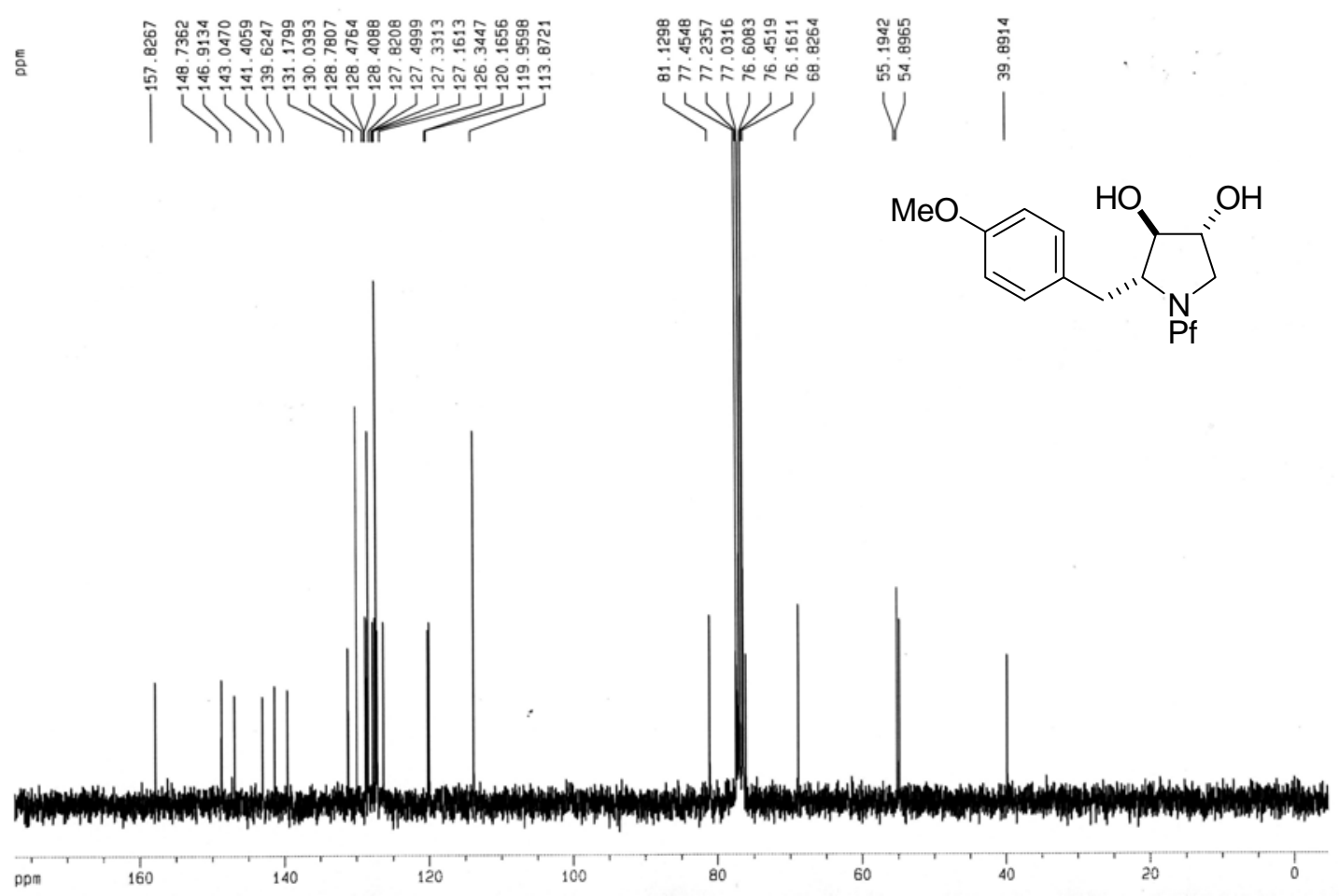

Spectra $17 .{ }^{13} \mathrm{C}$ NMR of $(\mathbf{2} R, 3 \boldsymbol{R}, \mathbf{4 R})-(\boldsymbol{N}-\mathbf{9}$-phenylfluoren-9-yl)-deacetyl anisomycin (12) 


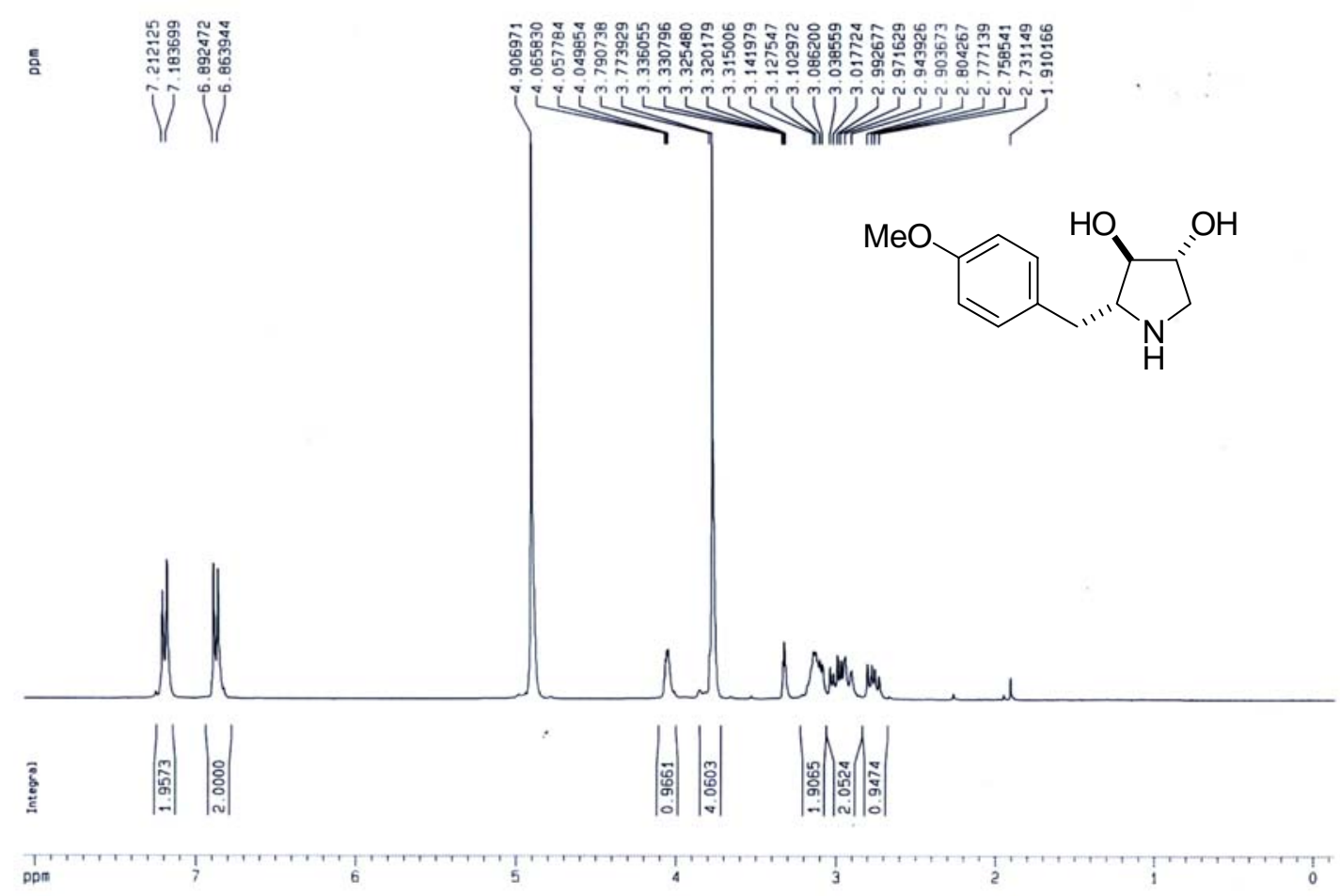

Spectra 18. ${ }^{1} \mathrm{H}$ NMR of (2R, 3R, $\left.4 \boldsymbol{R}\right)$-Deacetylanisomycin (3)

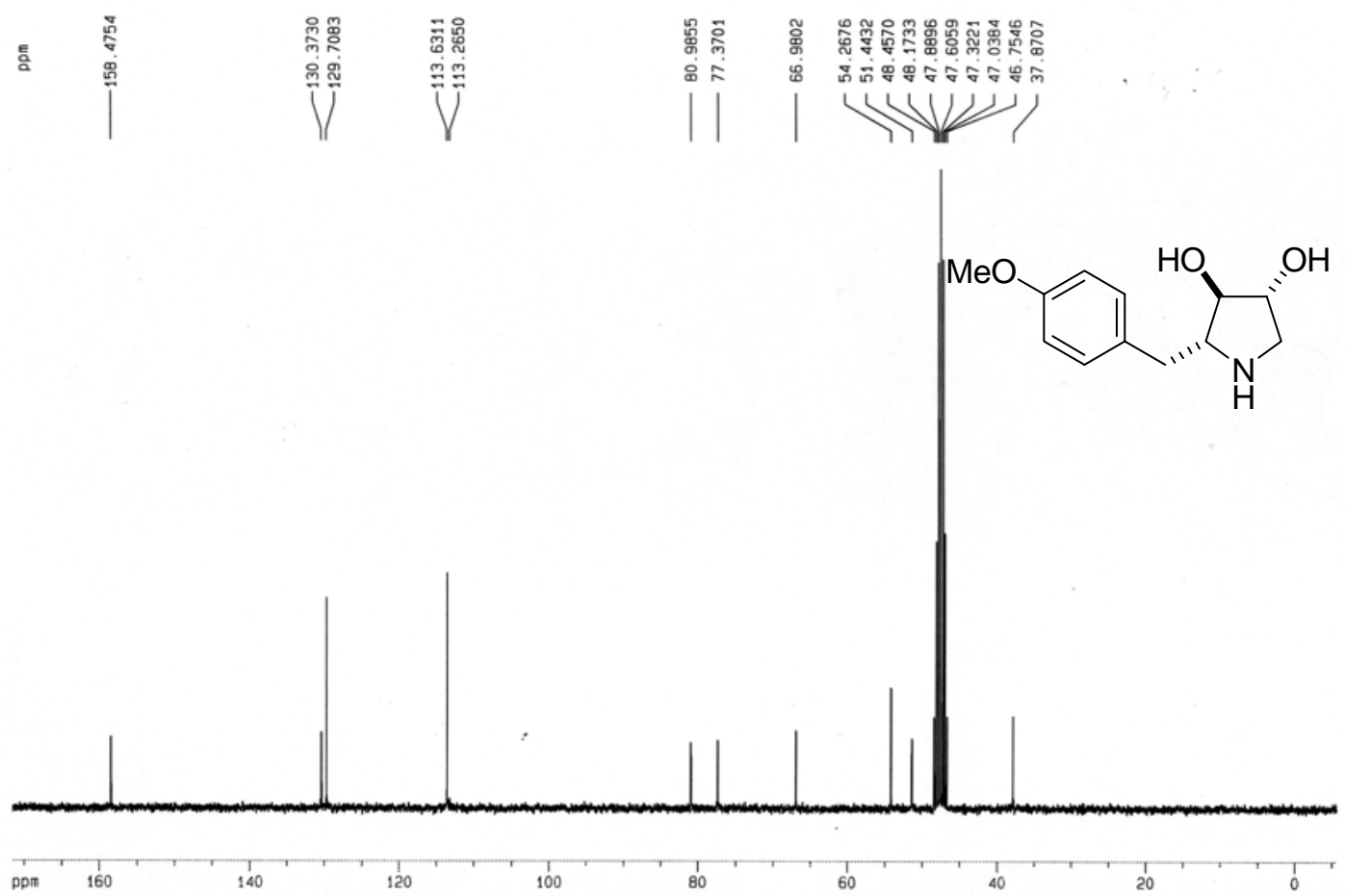

Spectra 19. ${ }^{1} \mathrm{H}$ NMR of $(\mathbf{2} \boldsymbol{R}, \mathbf{3} \boldsymbol{R}, \mathbf{4 R})$-Deacetylanisomycin (3) 


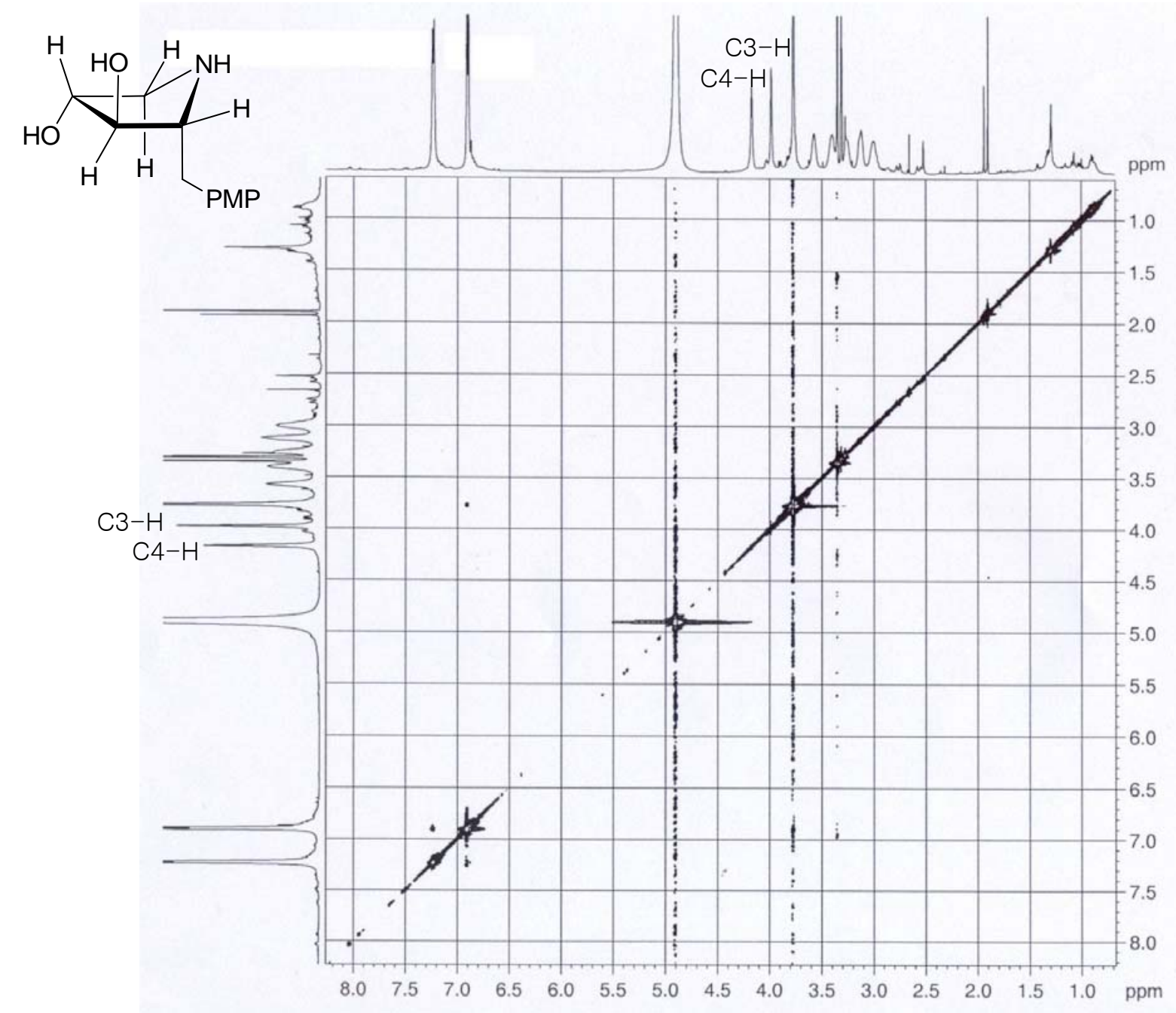

Spectra 20. NOESY of $(\mathbf{2} \boldsymbol{R}, \mathbf{3} \boldsymbol{R}, \mathbf{4 R})$-Deacetylanisomycin (3) 


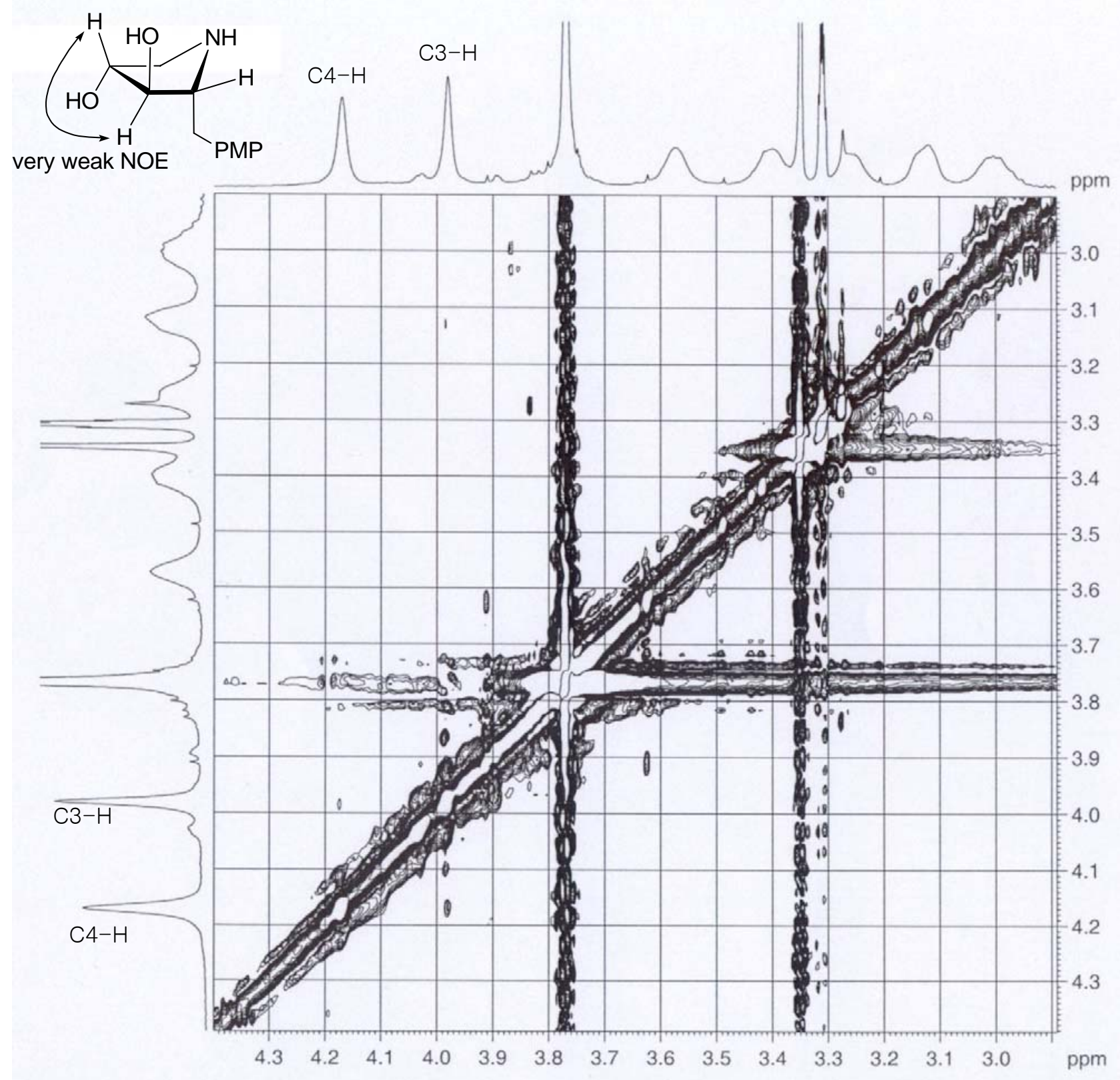

Spectra 21. Expended NOESY of (2R, 3R, 4R)-Deacetylanisomycin (3) 


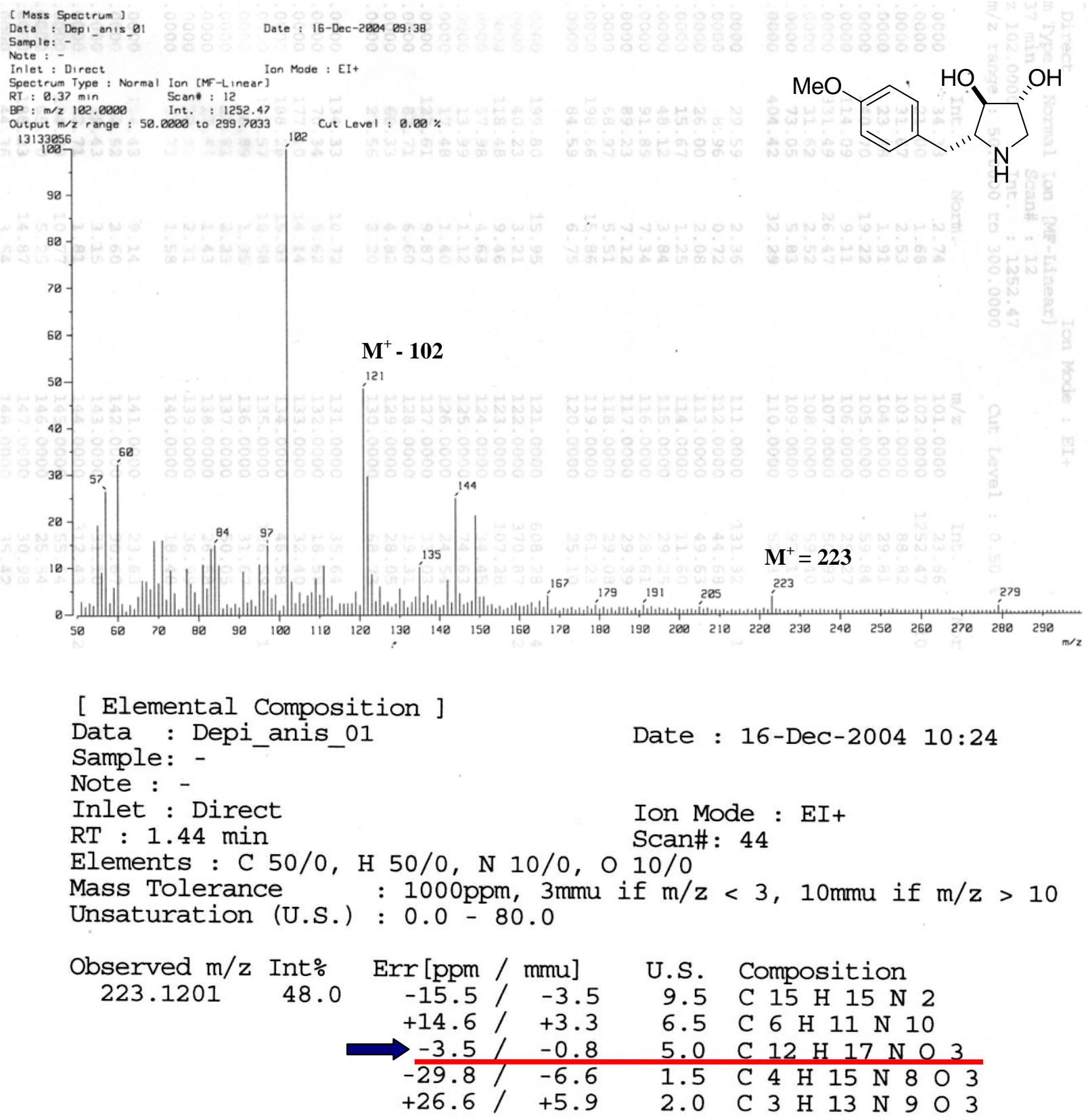

Spectra 22. EI mass \& HR mass of (2R, 3R, $4 R)$-Deacetylanisomycin (3) 


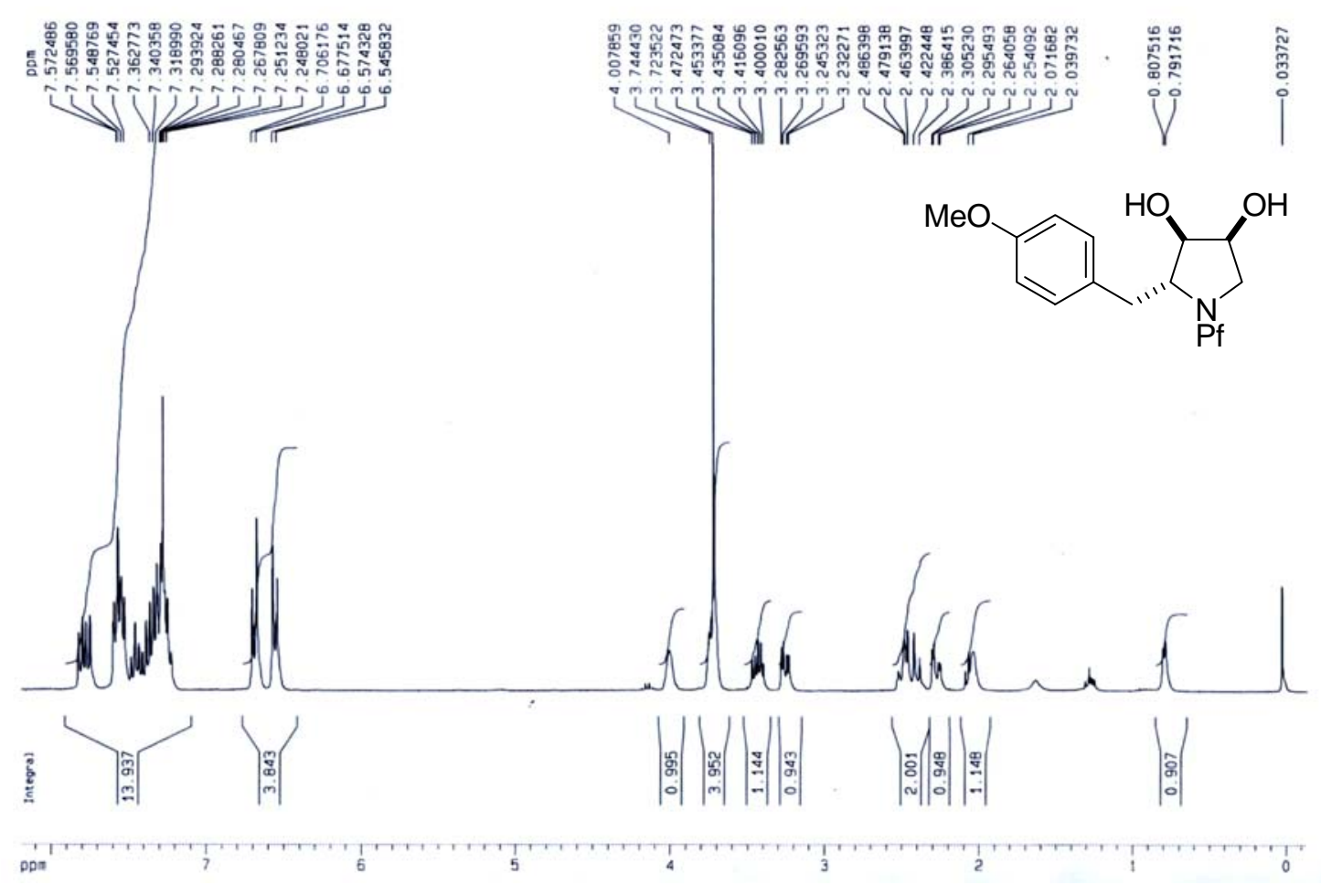

Spectra 23. ${ }^{1} \mathrm{H}$ NMR of $(\mathbf{2} R, \mathbf{3 R}, \mathbf{4 S})-(\boldsymbol{N}-\mathbf{9}-$ Phenylfluoren-9-yl) deacetyl anisomycin (14)

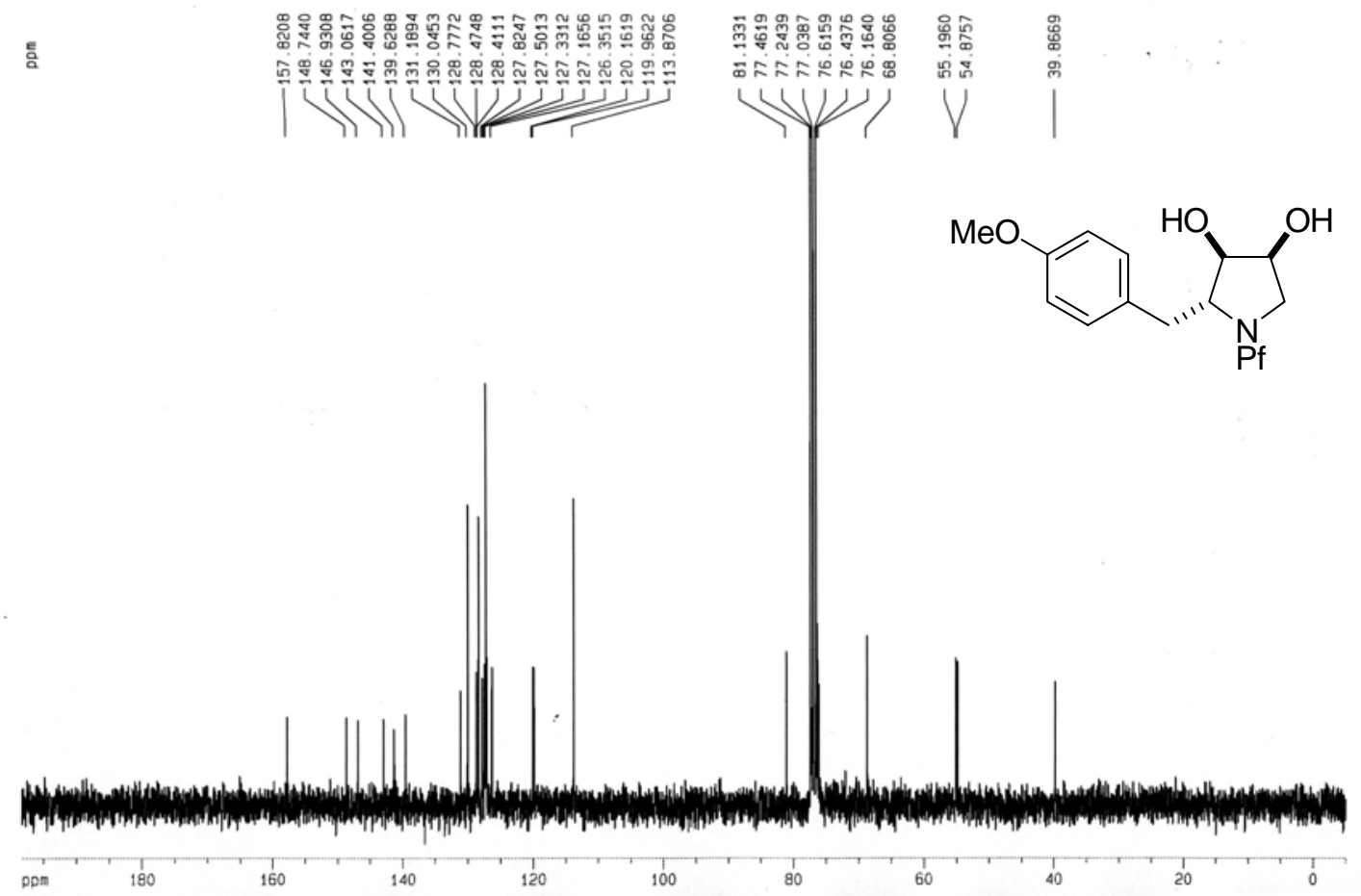

Spectra $24 .{ }^{13} \mathrm{C}$ NMR of $(\mathbf{2} R, \mathbf{3 R}, \mathbf{4 S})-(\boldsymbol{N}-\mathbf{9}-$ Phenylfluoren-9-yl) deacetyl anisomycin (14) 


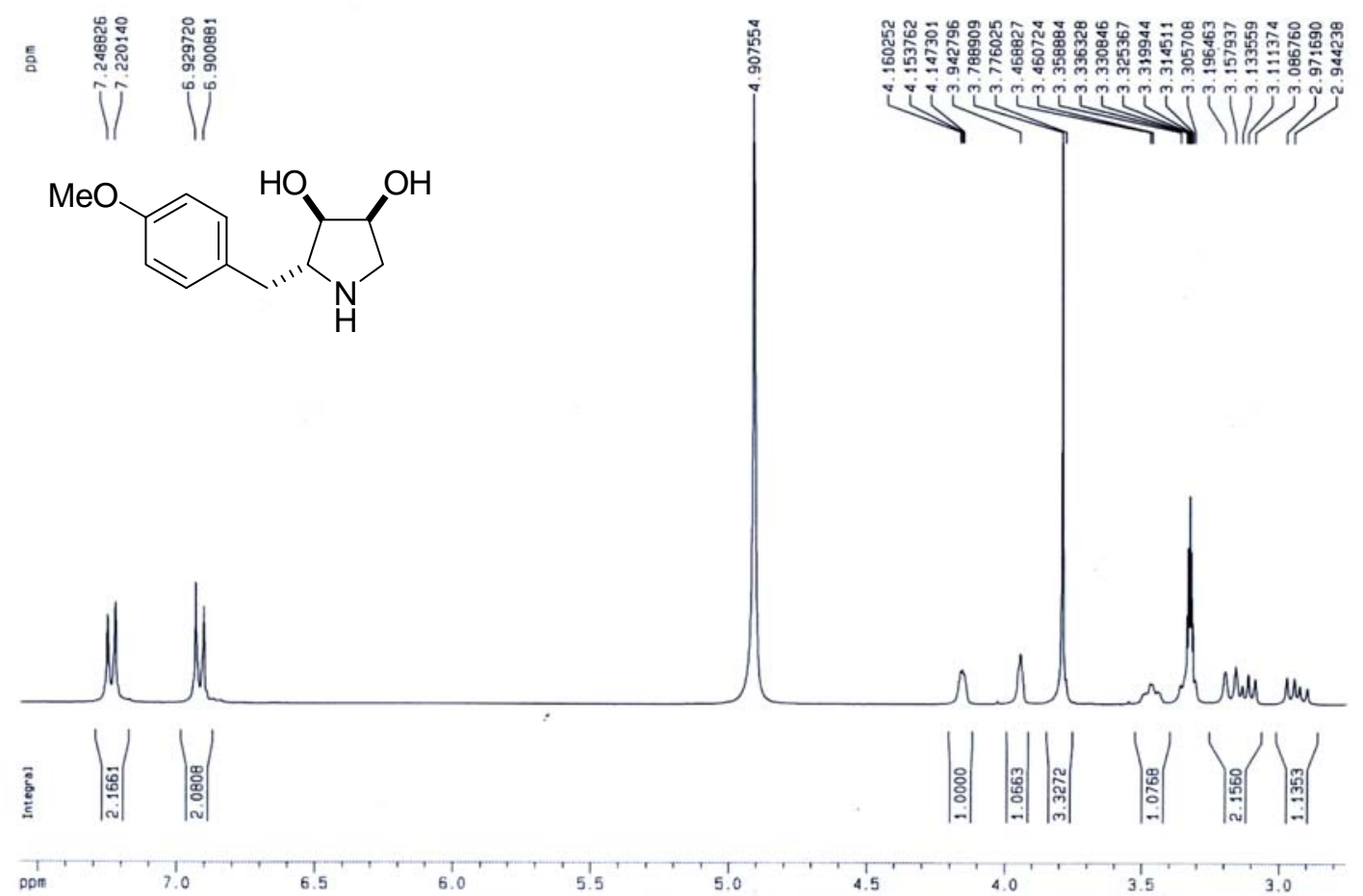

Spectra 25. ${ }^{1} \mathrm{H}$ NMR of (2R, 3R, 4S)-Deacetylanisomycin (4)

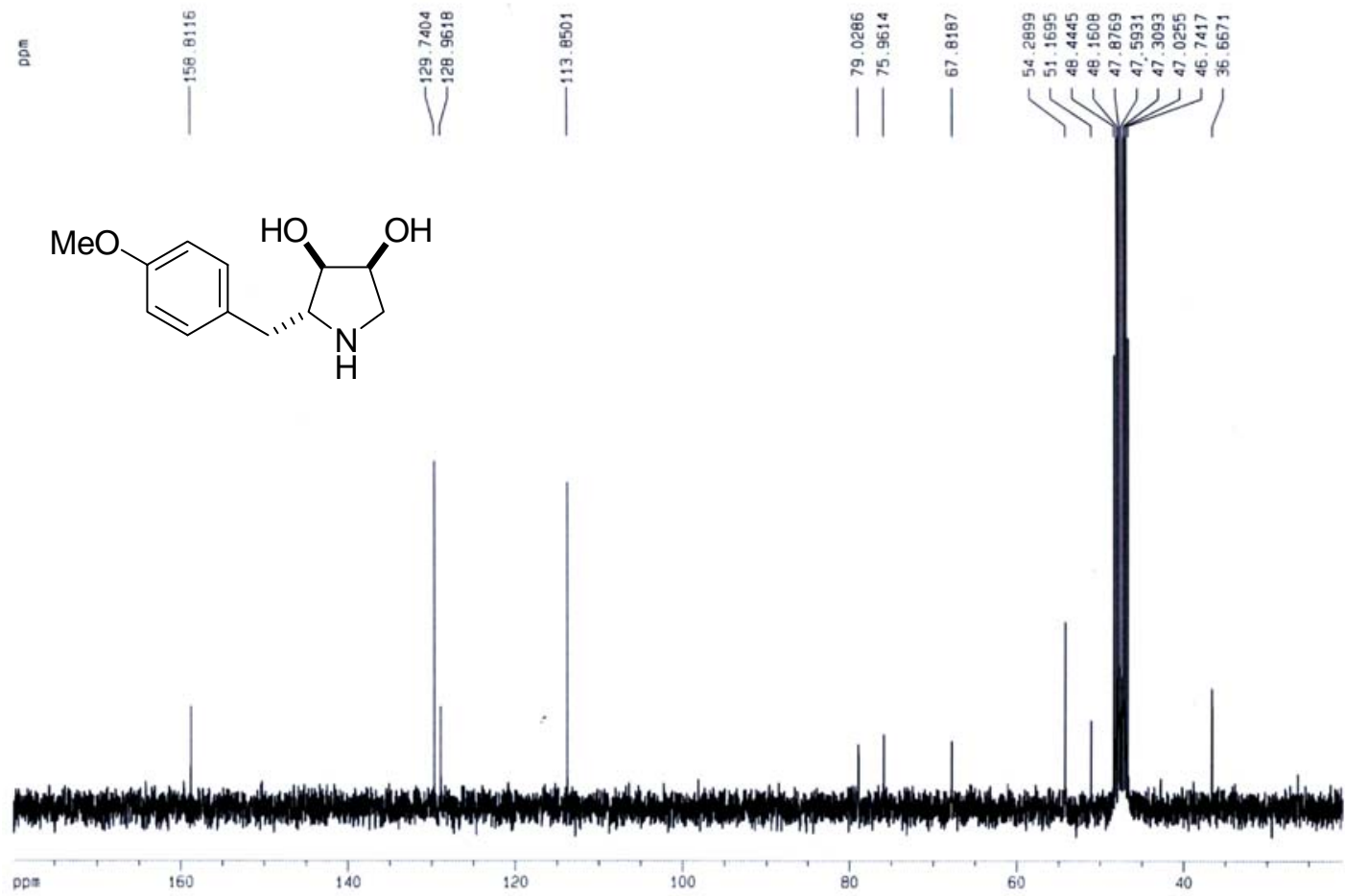

Spectra 26. ${ }^{13} \mathrm{C}$ NMR of (2R, 3R, 4S)-Deacetylanisomycin (4) 


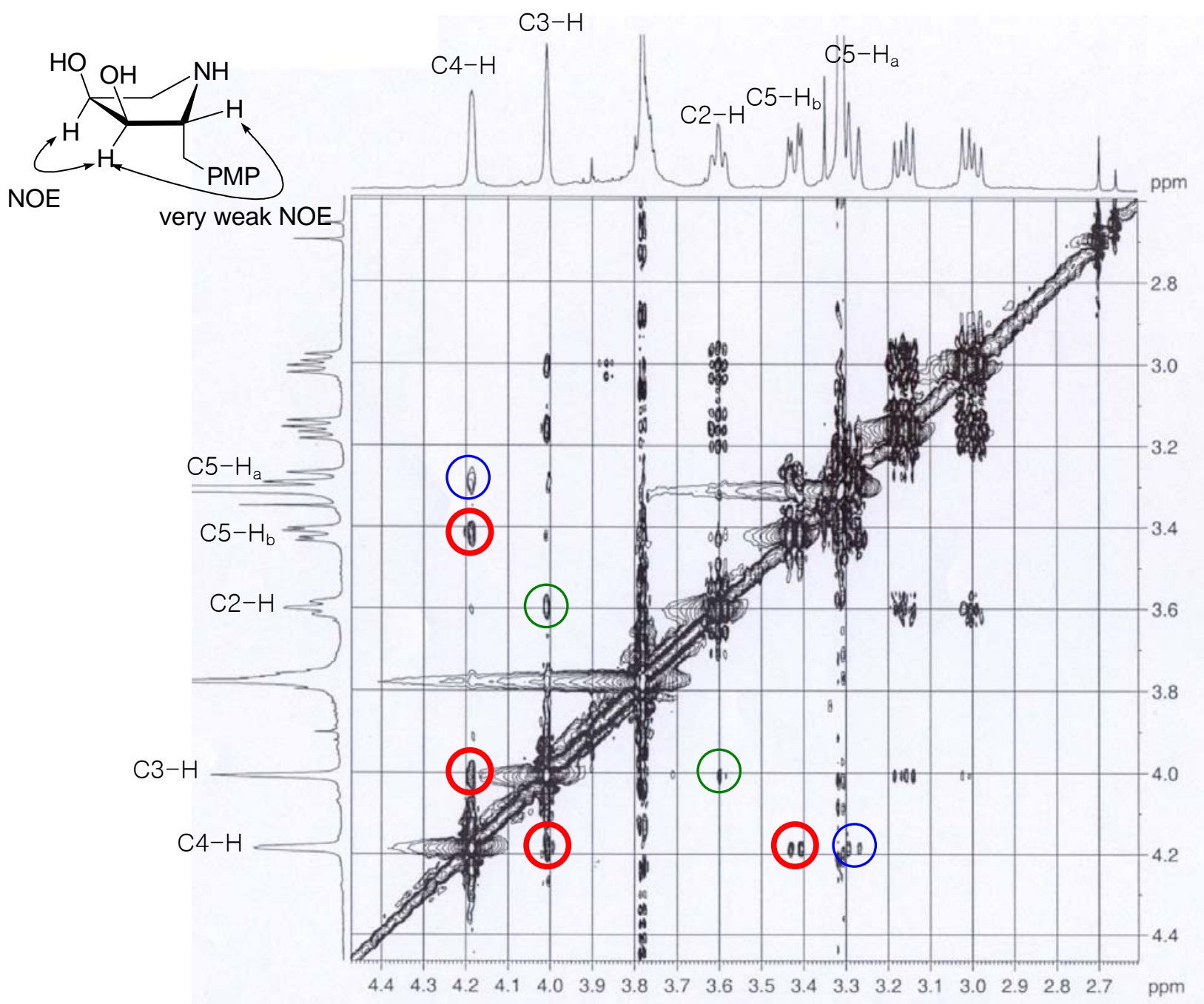

Spectra 27. NOESY of $(2 R, 3 R, 4 S)$-Deacetylanisomycin (4) 


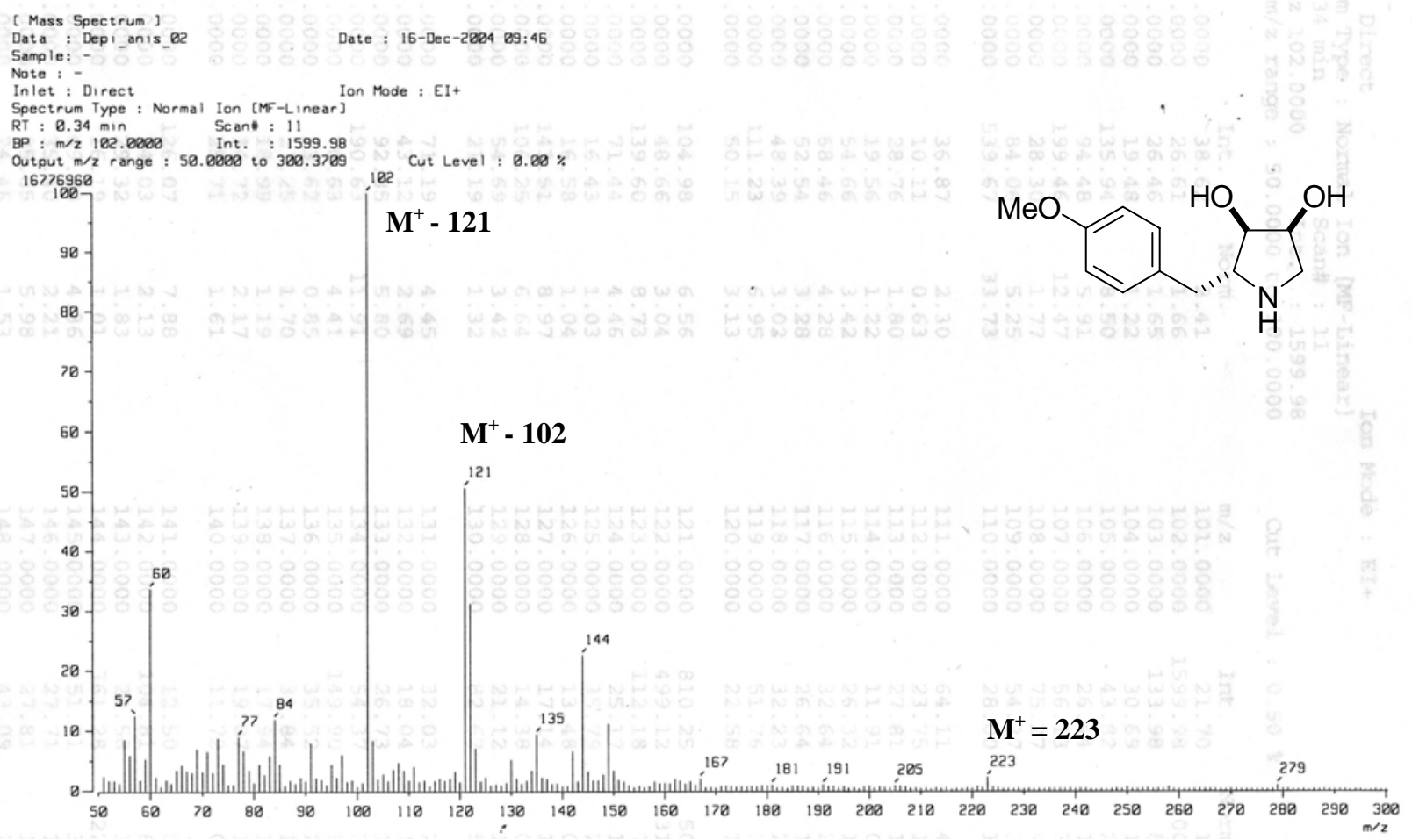

[ Elemental Composition ]

Data : Depi_anis_02

Date : 16-Dec-2004 10:37

Page: 1

Sample:

Note : -

Inlet : Direct

RT : $0.70 \mathrm{~min}$

Ion Mode : EI+

Elements : C 50/0, H 50/0, N $10 / 0$, O 10/0

Mass Tolerance : 1000ppm, 3mmu if $\mathrm{m} / \mathrm{z}<3,10 \mathrm{mmu}$ if $\mathrm{m} / \mathrm{z}>10$

Unsaturation (U.S.) : $0.0-80.0$

\begin{tabular}{|c|c|c|c|c|c|}
\hline $\begin{array}{c}\text { Observed m/z } \\
223.1205\end{array}$ & $\begin{array}{l}\text { Int } \% \\
100.0\end{array}$ & $\begin{array}{r}\text { Err [ppm / } \\
-13.6 \% \\
+10.4 \% \\
+4.4 \% \\
\Rightarrow-1.6 / \\
-27.9 \% \\
+28.5 /\end{array}$ & $\begin{array}{l}\mathrm{mmu}] \\
-3.0 \\
+2.3 \\
+1.0 \\
-0.4 \\
-6.2 \\
+6.3\end{array}$ & $\begin{array}{r}\text { U.S. } \\
9.5 \\
6.0 \\
5.5 \\
5.0 \\
1.5 \\
2.0\end{array}$ & 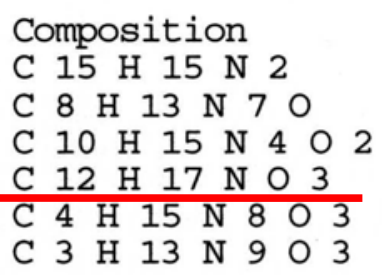 \\
\hline
\end{tabular}

Spectra 28. EI mass \& HR mass of (2R, 3R, 4S)-Deacetylanisomycin (4) 


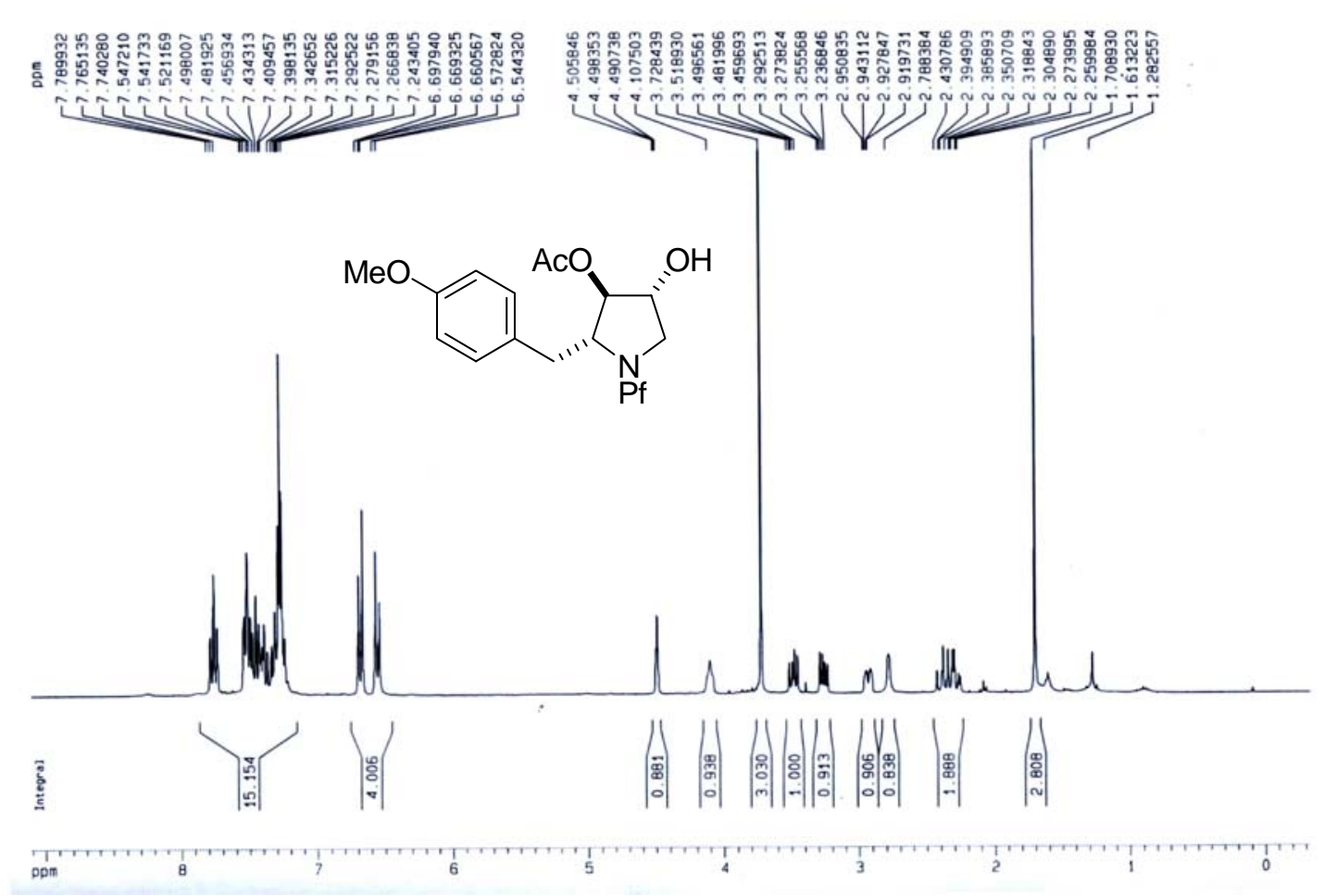

Spectra 29. ${ }^{1} \mathrm{H}$ NMR of (2R, 3R, 4R)-( $N$-9-phenylfluoren-9-yl) anisomycin (15)

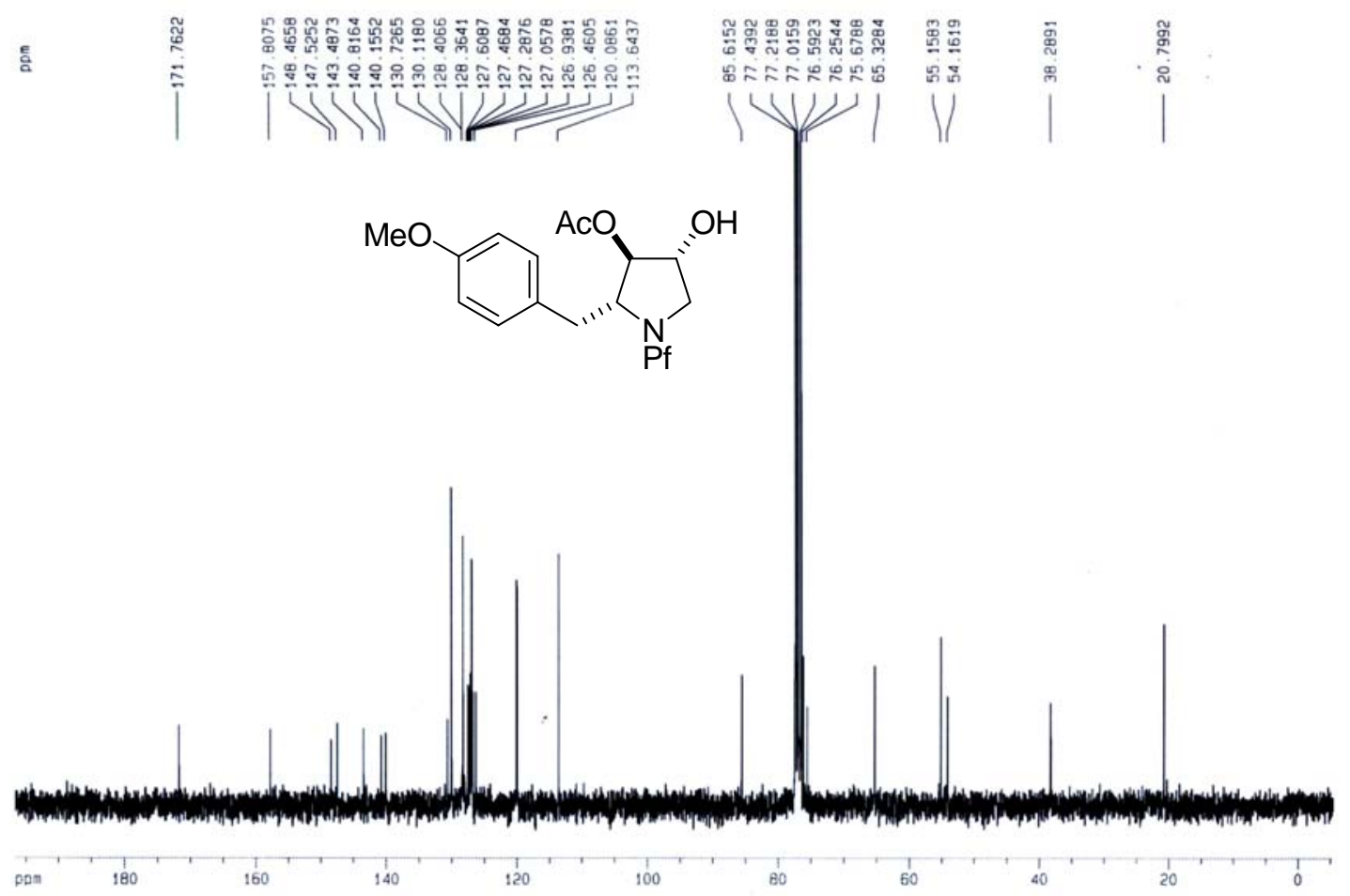

Spectra $30 .{ }^{13} \mathrm{C}$ NMR of $(\mathbf{2} \boldsymbol{R}, \mathbf{3 R}, \mathbf{4 R})-(\boldsymbol{N}-\mathbf{9}$-phenylfluoren-9-yl) anisomycin (15) 


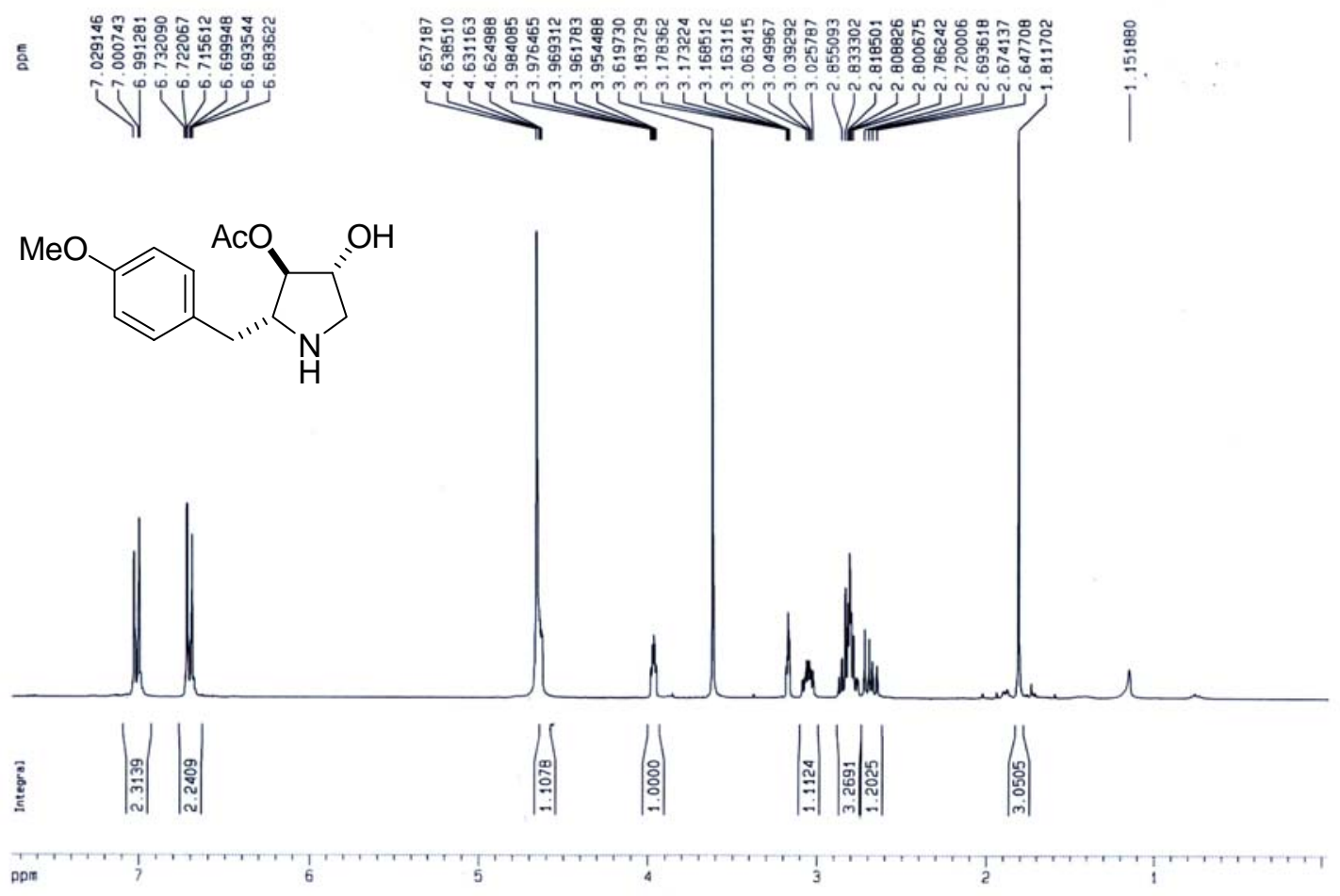

Spectra 31. ${ }^{1} \mathrm{H}$ NMR of (2R, 3R, $\left.4 \boldsymbol{R}\right)$-anisomycin (2)

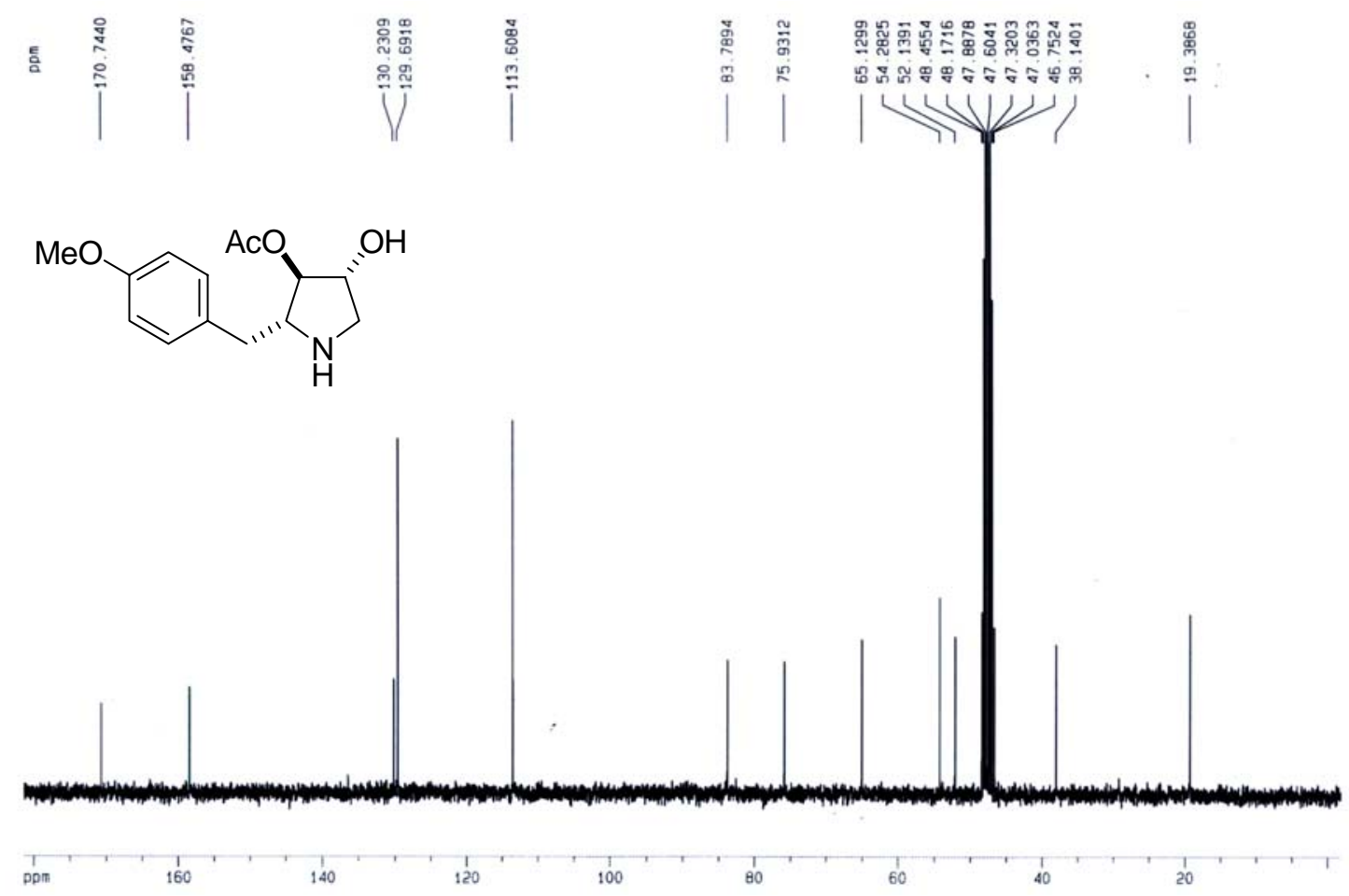

Spectra 32. ${ }^{13} \mathrm{C}$ NMR of (2R, 3R, 4R)-anisomycin (2) 

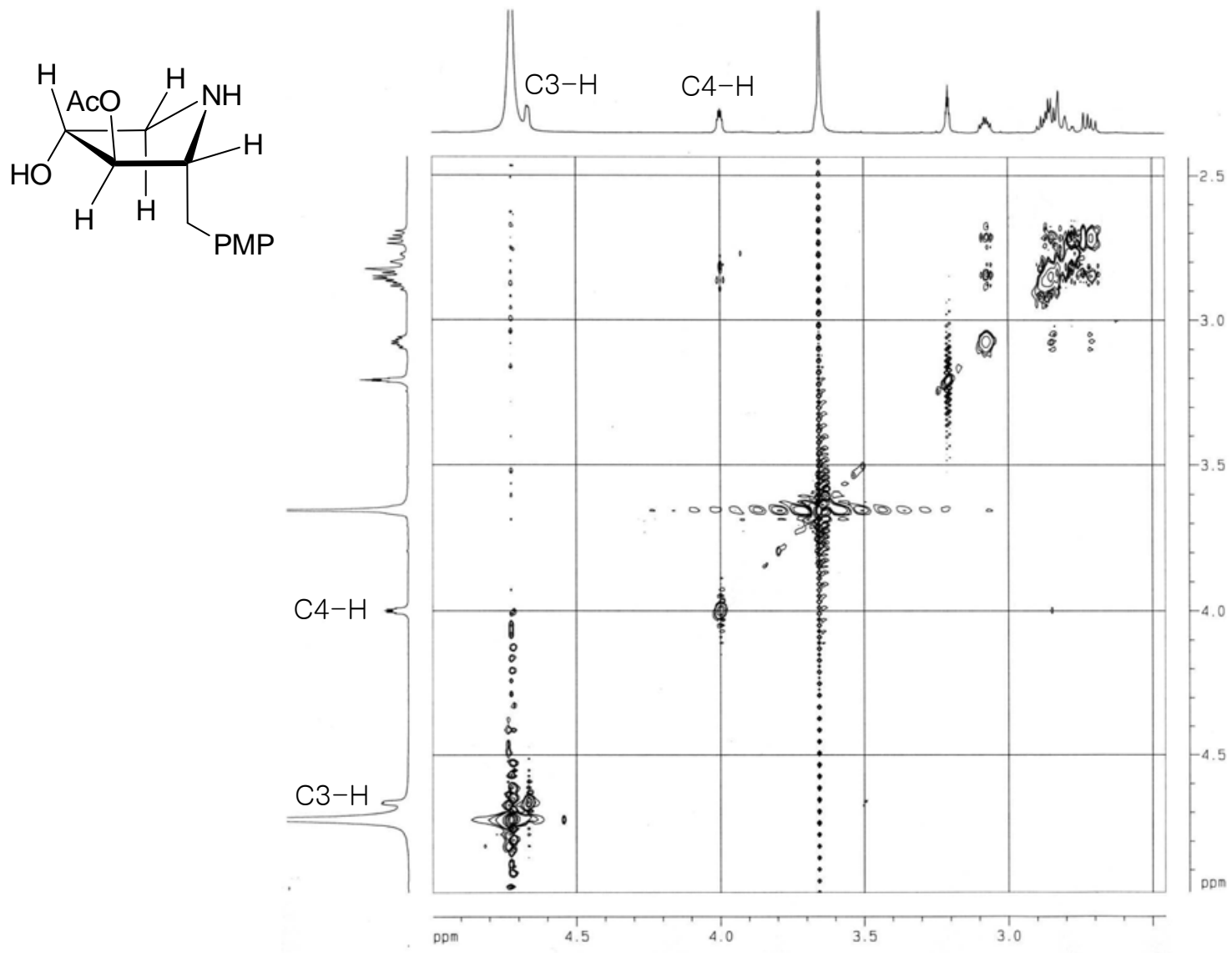

Spectra 33. NOESY of $(\mathbf{2} \boldsymbol{R}, \mathbf{3 R}, \mathbf{4 R})$-anisomycin (2). 


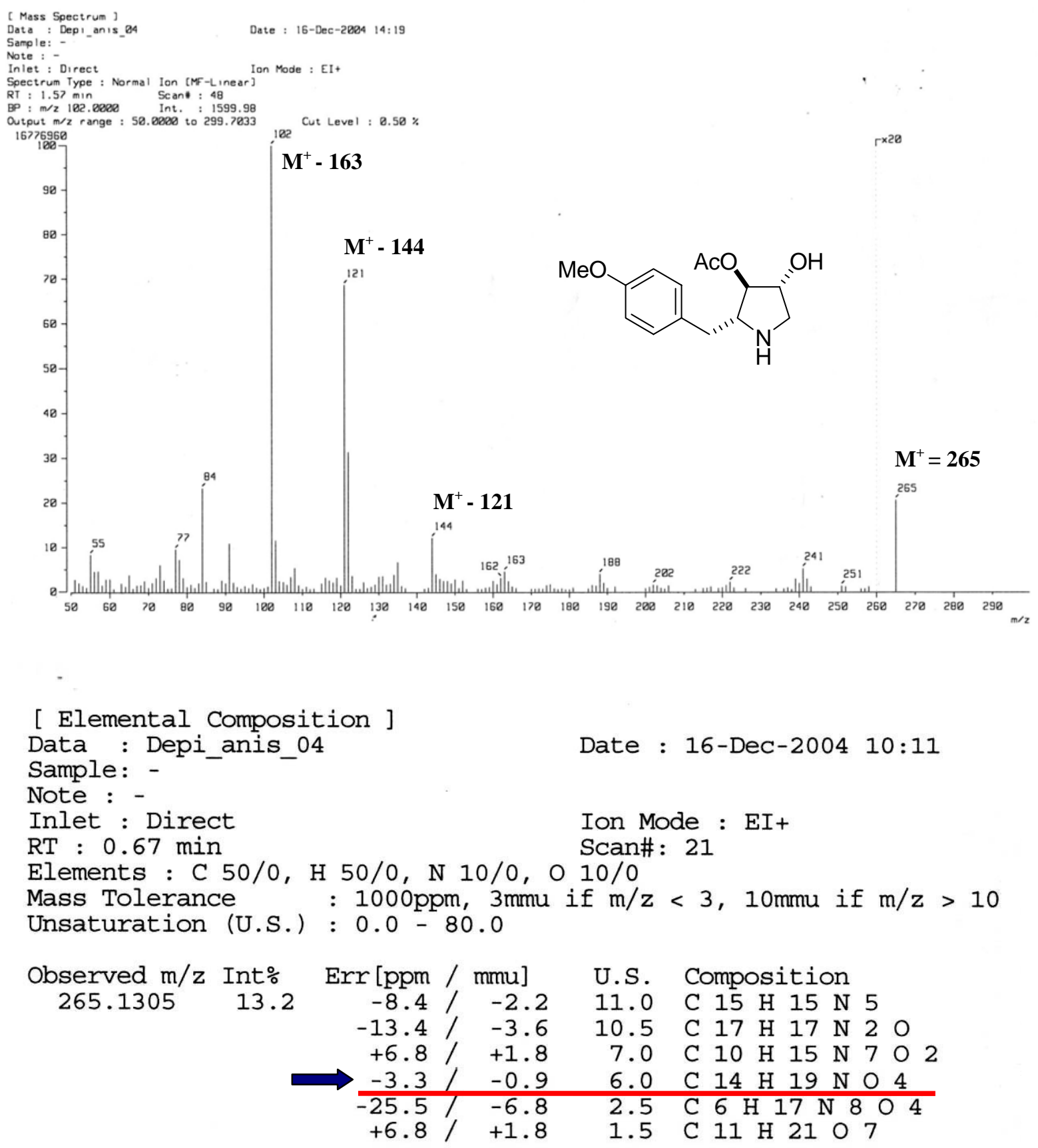

Spectra 34. EI mass \& HR mass of (2R, 3R, $4 \boldsymbol{R})$-anisomycin (2) 


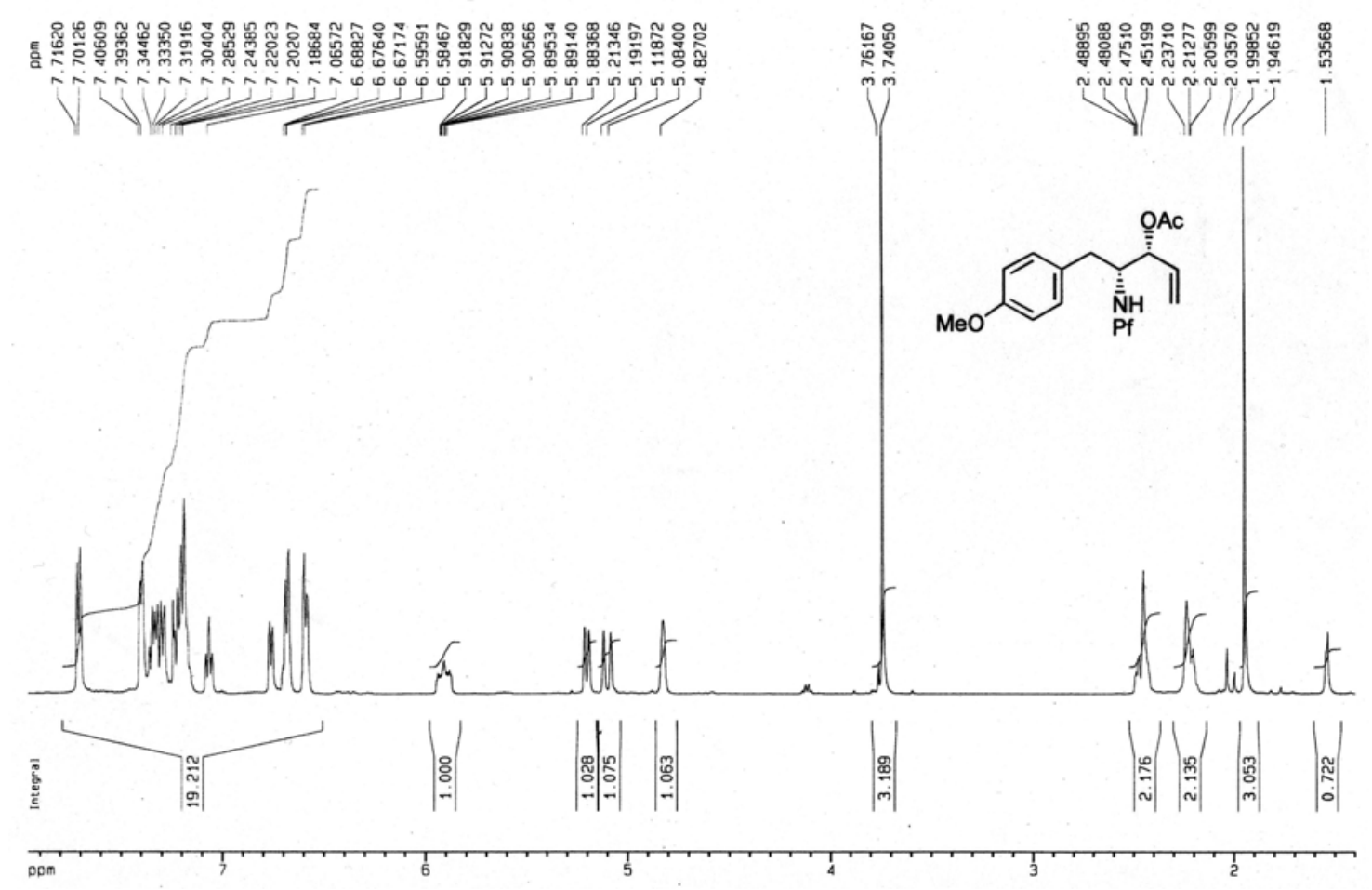

Spectra 35. $\quad{ }^{1} \mathrm{H} \quad$ NMR of $\quad(3 \boldsymbol{R}, \quad \mathbf{4 R})-\mathbf{4}-(\boldsymbol{N}-\mathbf{9}-$ phenylfluoren-9-yl)amino-3-acetoxy-5-(pmethoxyphenyl)-1-pentene (16)

咅

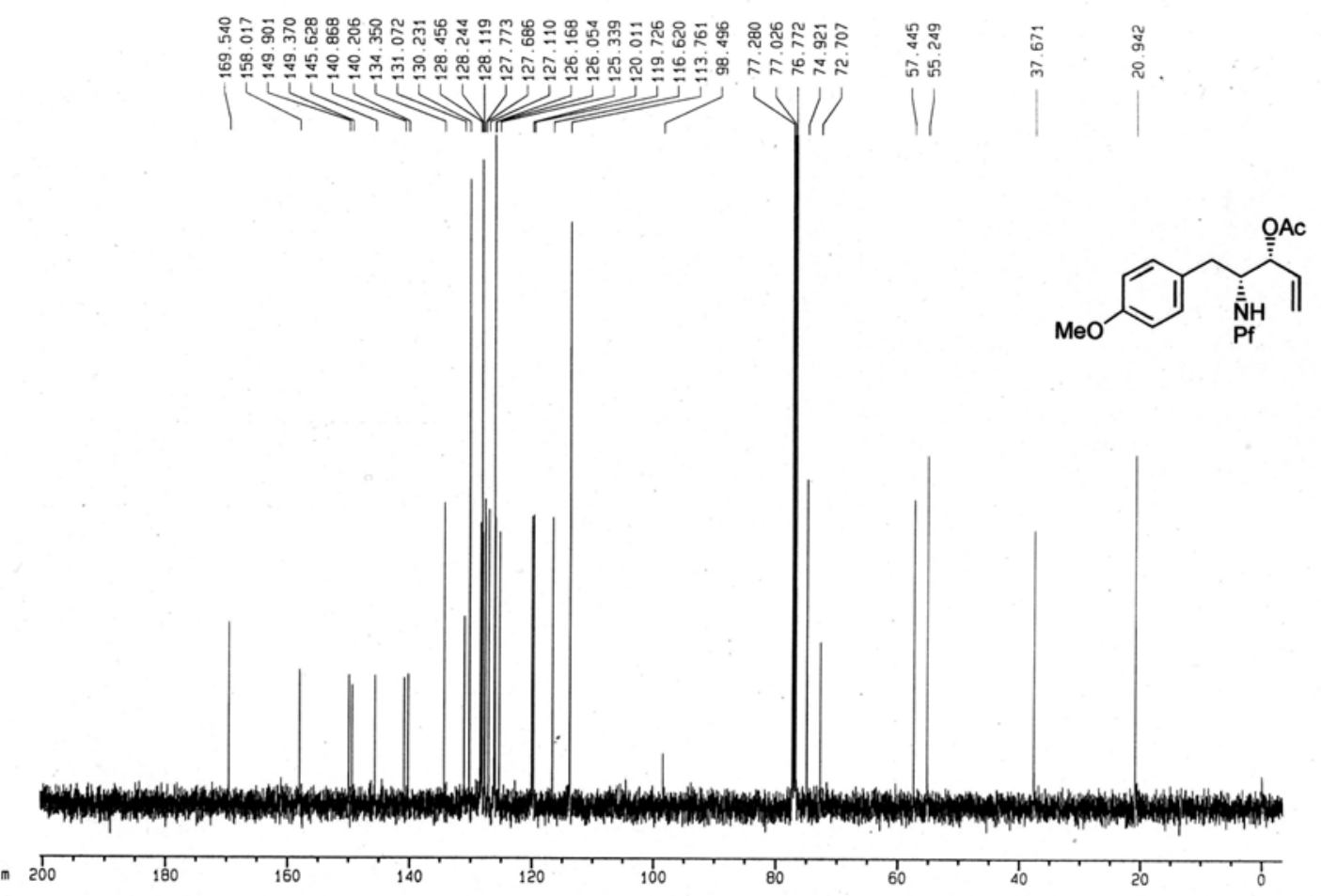

Spectra 36. ${ }^{13} \mathrm{C} \quad \mathrm{NMR} \quad$ of $\quad(3 \boldsymbol{R}, \quad \mathbf{4 R})-\mathbf{4}-(\boldsymbol{N}-\mathbf{9}-$ phenylfluoren-9-yl)amino-3-acetoxy-5-(pmethoxyphenyl)-1-pentene (16) 


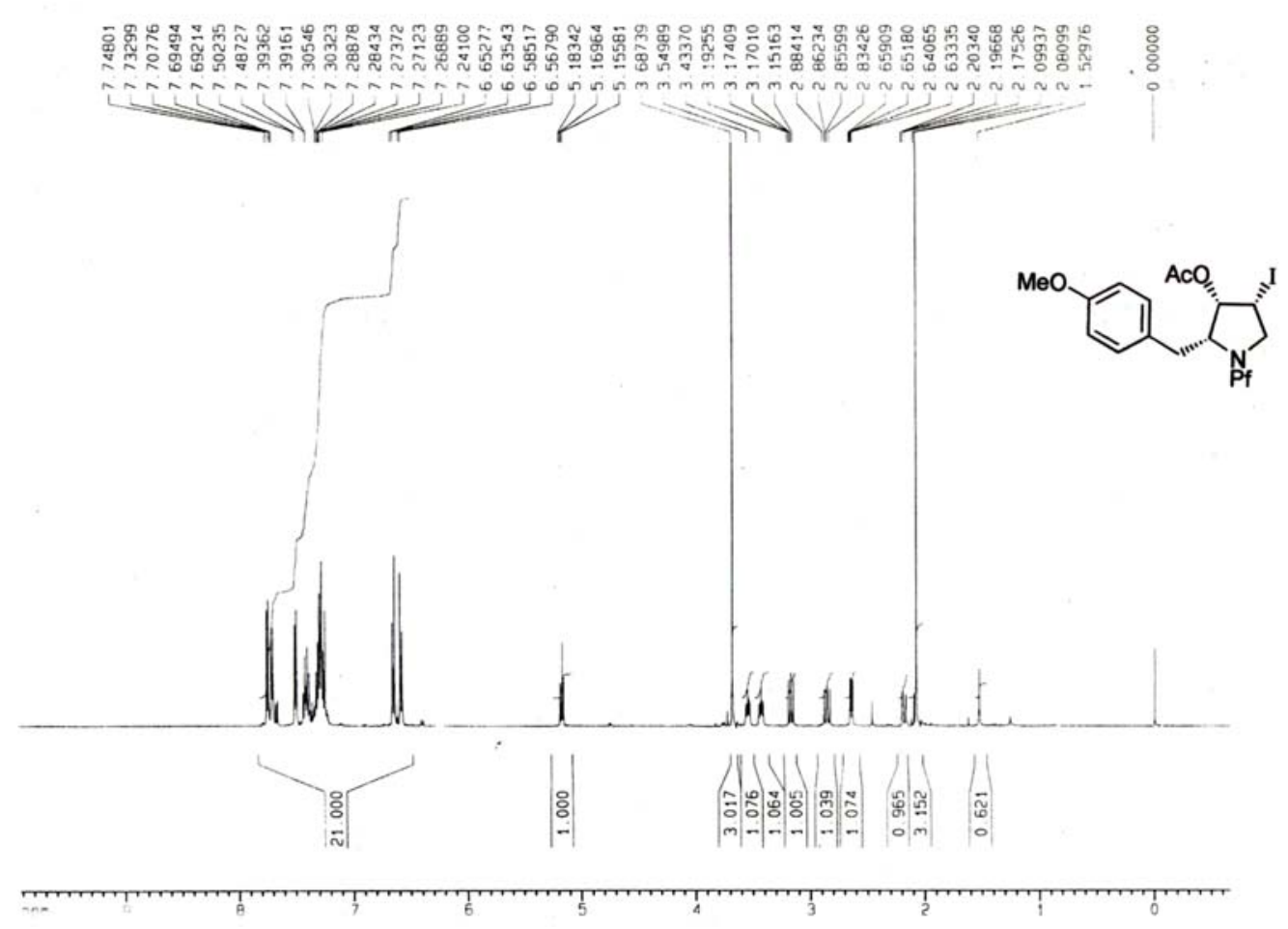

Spectra 37. ${ }^{1} \mathrm{H} \quad \mathrm{NMR}$ of $(\mathbf{2} \boldsymbol{R}, \quad 3 S, \quad 4 R)-4-(N-9-p h e n y l f l u o r e n-9-y l)-3-a c e t o x y-4-i o d o-2-(p-$ methoxybenzyl) pyrrolidine (17)

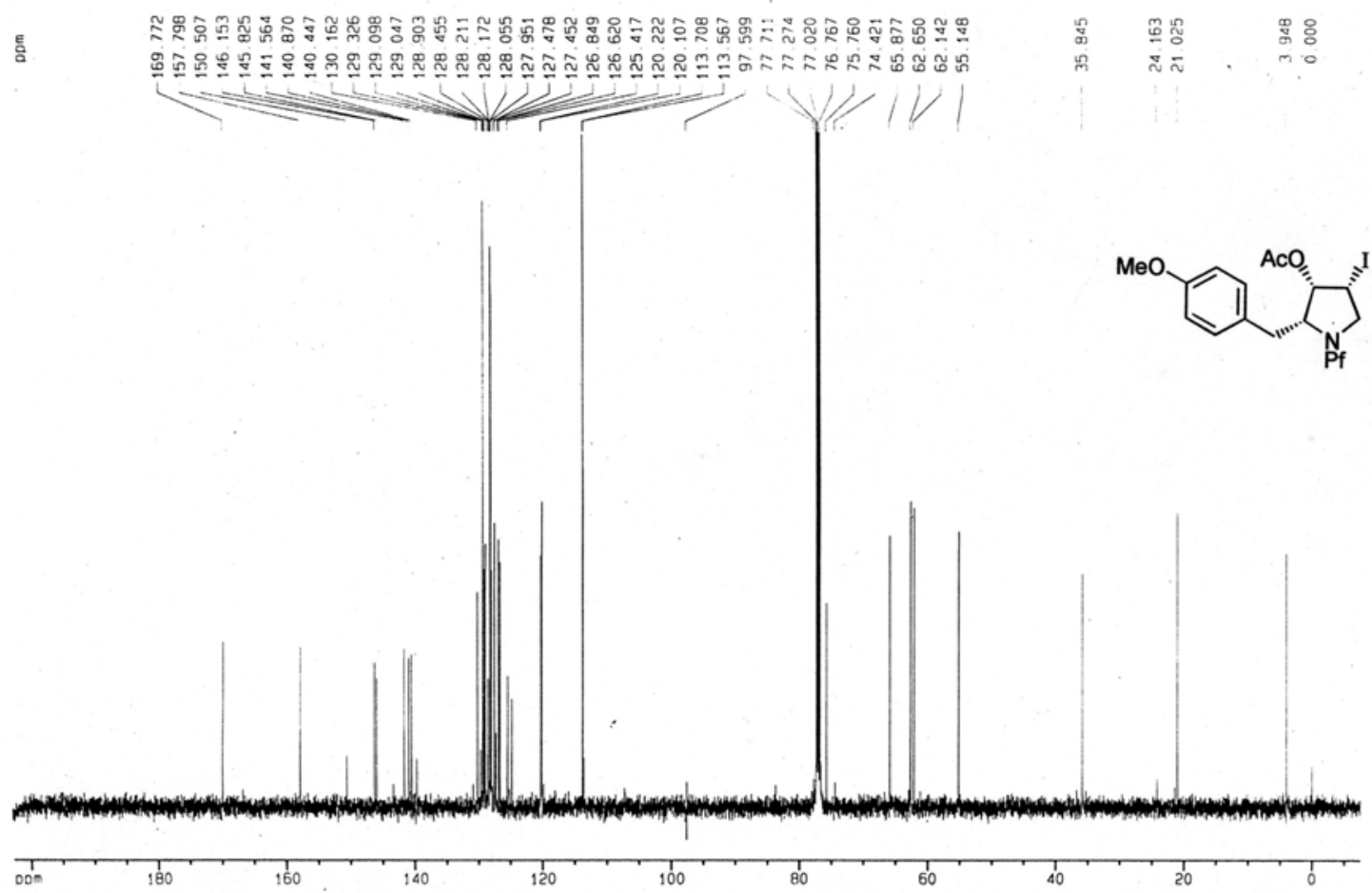

Spectra $38 .{ }^{13} \mathrm{C}$ NMR of $(\mathbf{2} R, \quad 3 S, \quad 4 R)-4-(N-9-p h e n y l f l u o r e n-9-y l)-3-a c e t o x y-4-i o d o-2-(p-$ methoxybenzyl) pyrrolidine (17) 


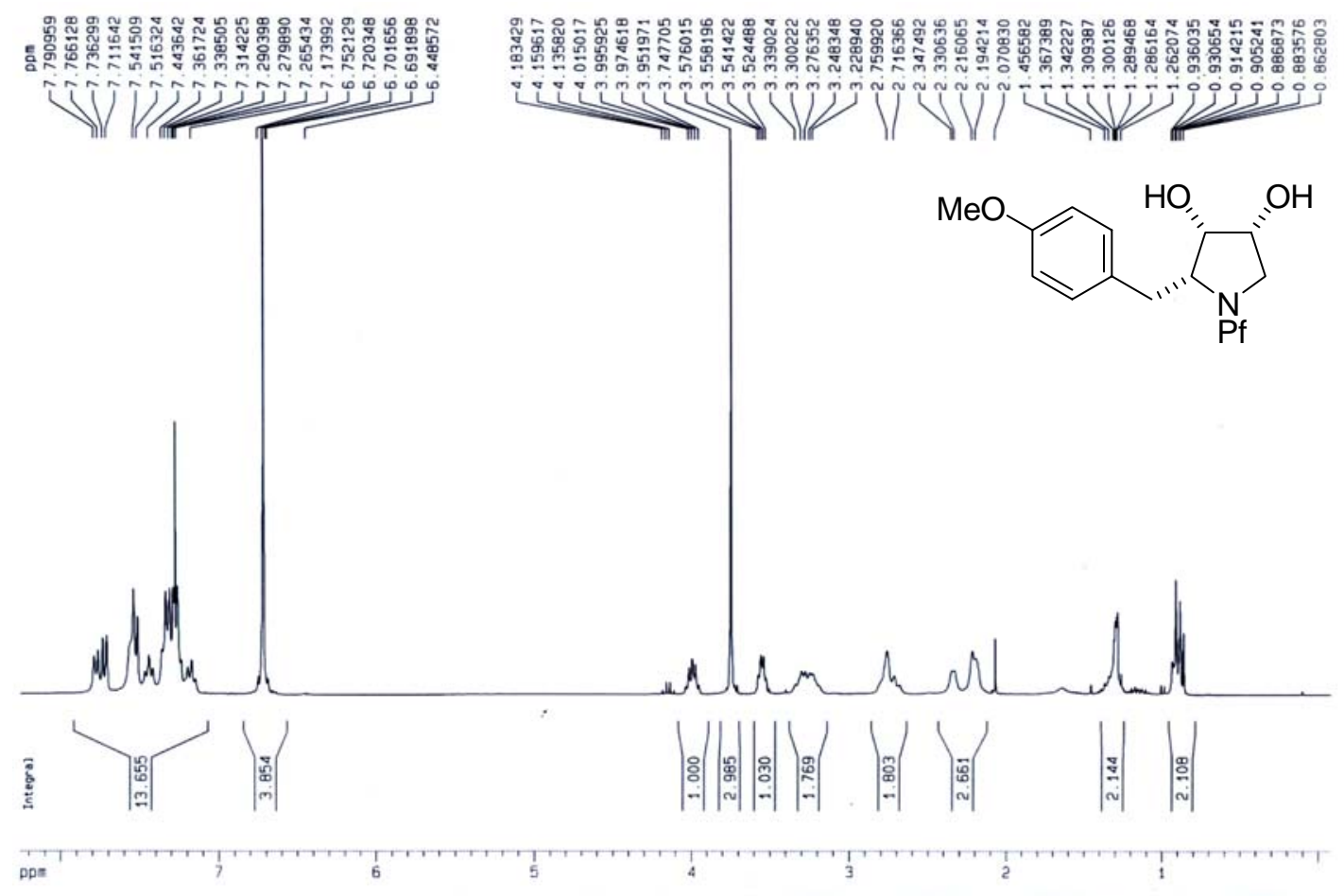

Spectra 39. ${ }^{1} \mathrm{H}$ NMR of (2R, 3S, 4R)-(N-9-Phenylfluoren-9-yl)-deacetyl anisomycin (19)

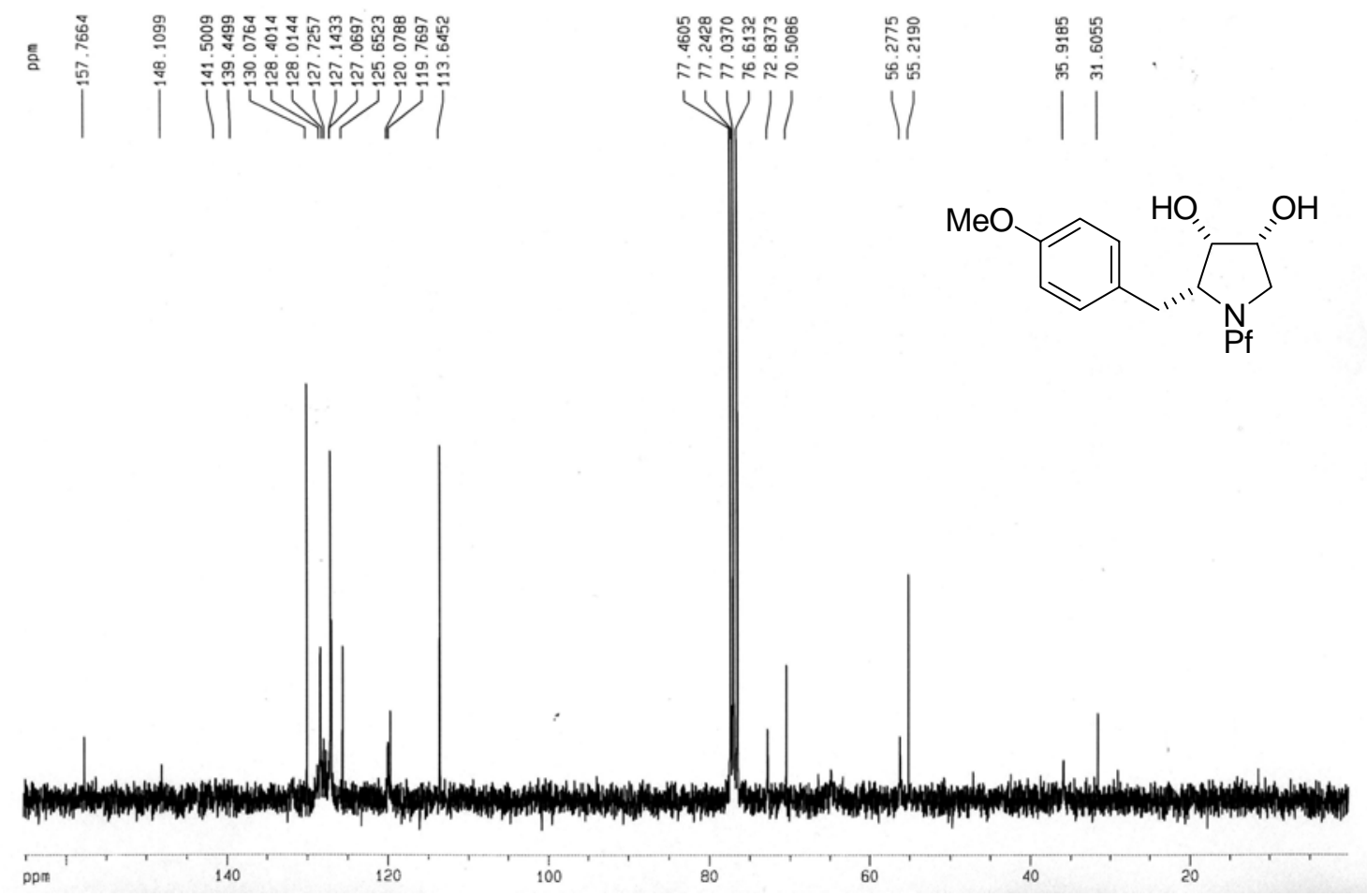

Spectra 40. ${ }^{13} \mathrm{C}$ NMR of (2R, 3S, 4R)-(N-9-Phenylfluoren-9-yl)-deacetyl anisomycin (19) 


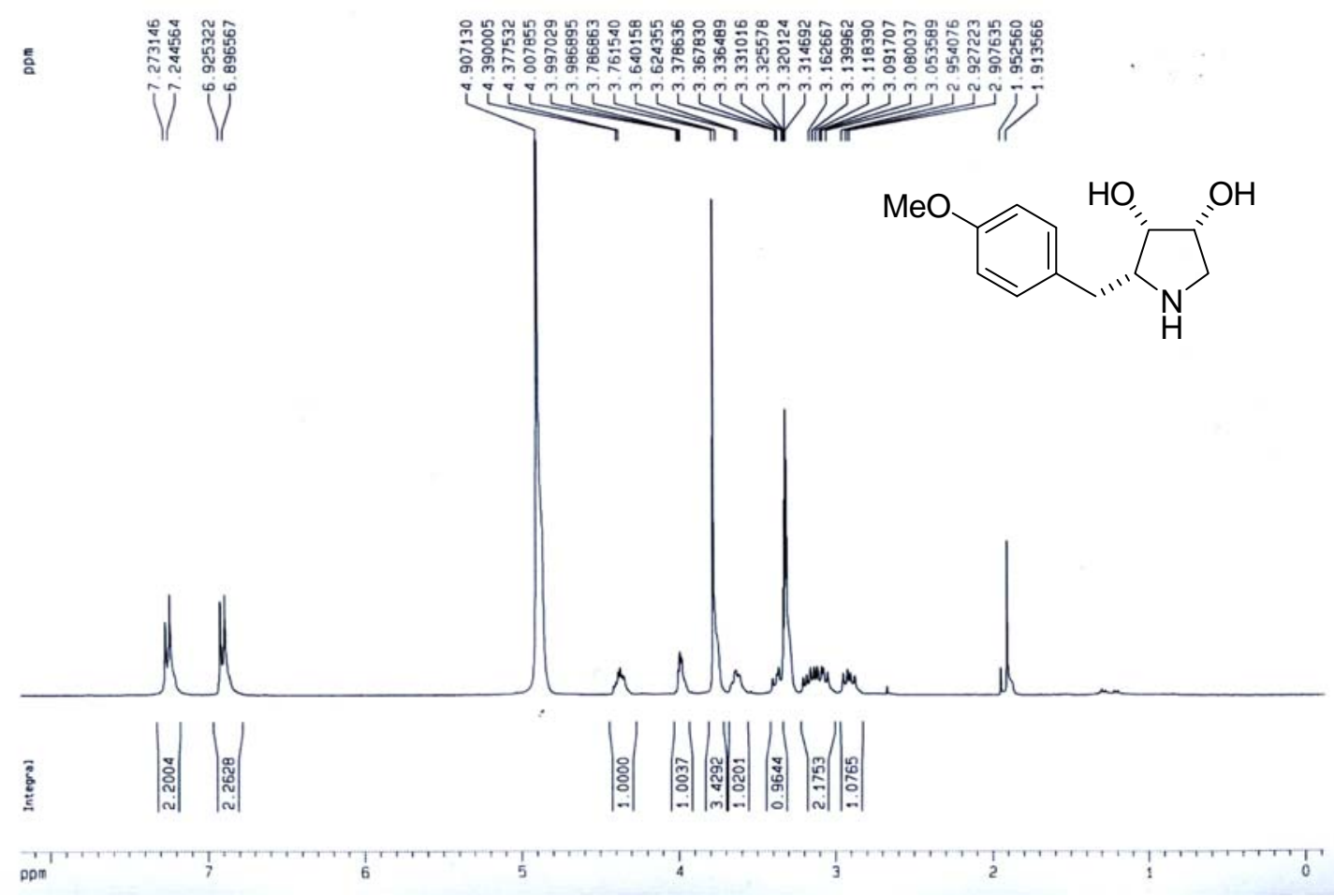

Spectra 41. ${ }^{1} \mathrm{H}$ NMR of (2R, 3S, 4R)-Deacetylanisomycin (5)<smiles>COc1ccc(C[C@@H]2NC[C@H](O)[C@H]2O)cc1</smiles>
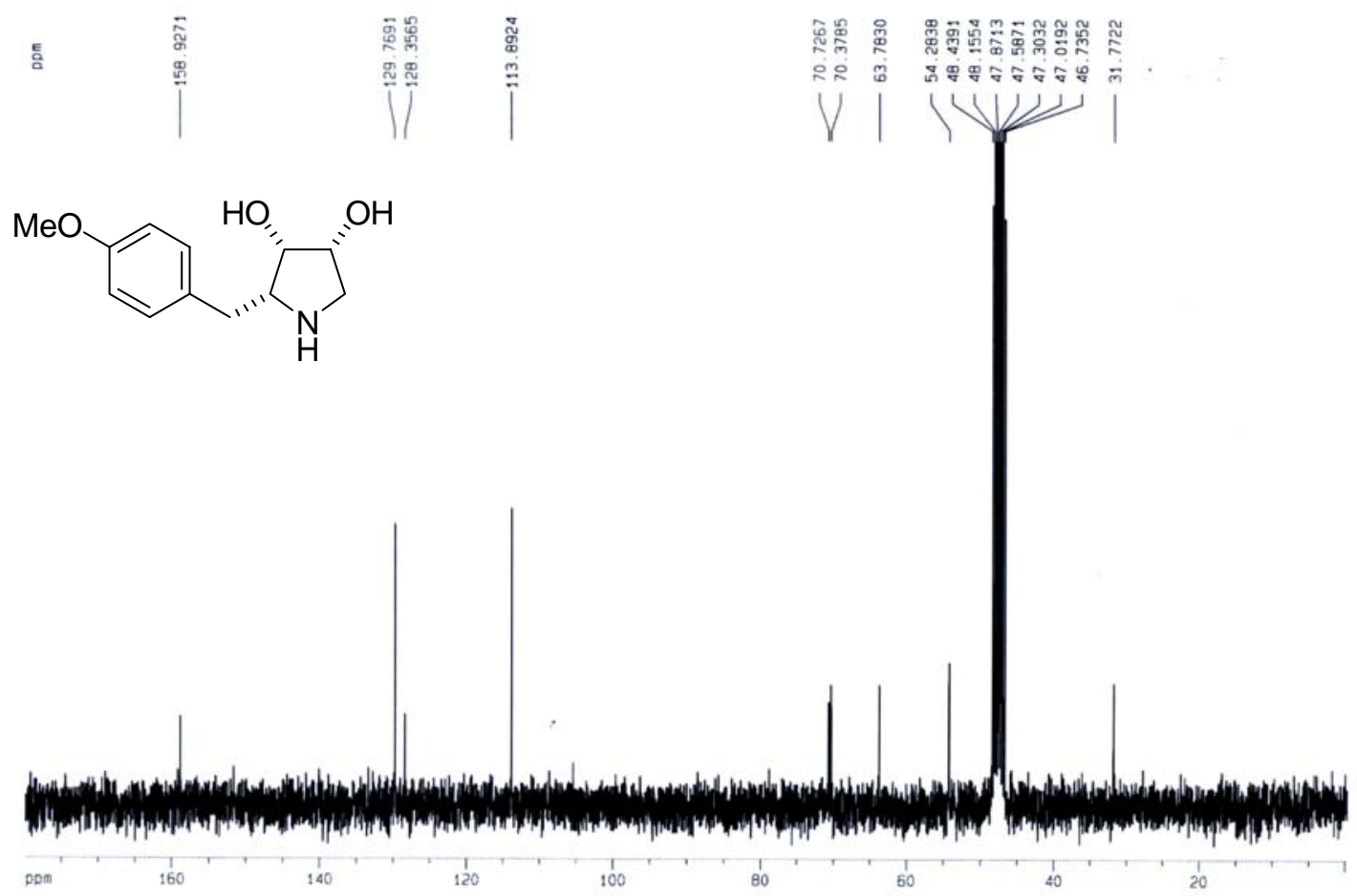

Spectra 42. ${ }^{13} \mathrm{C}$ NMR of (2R, 3S, 4R)-Deacetylanisomycin (5) 


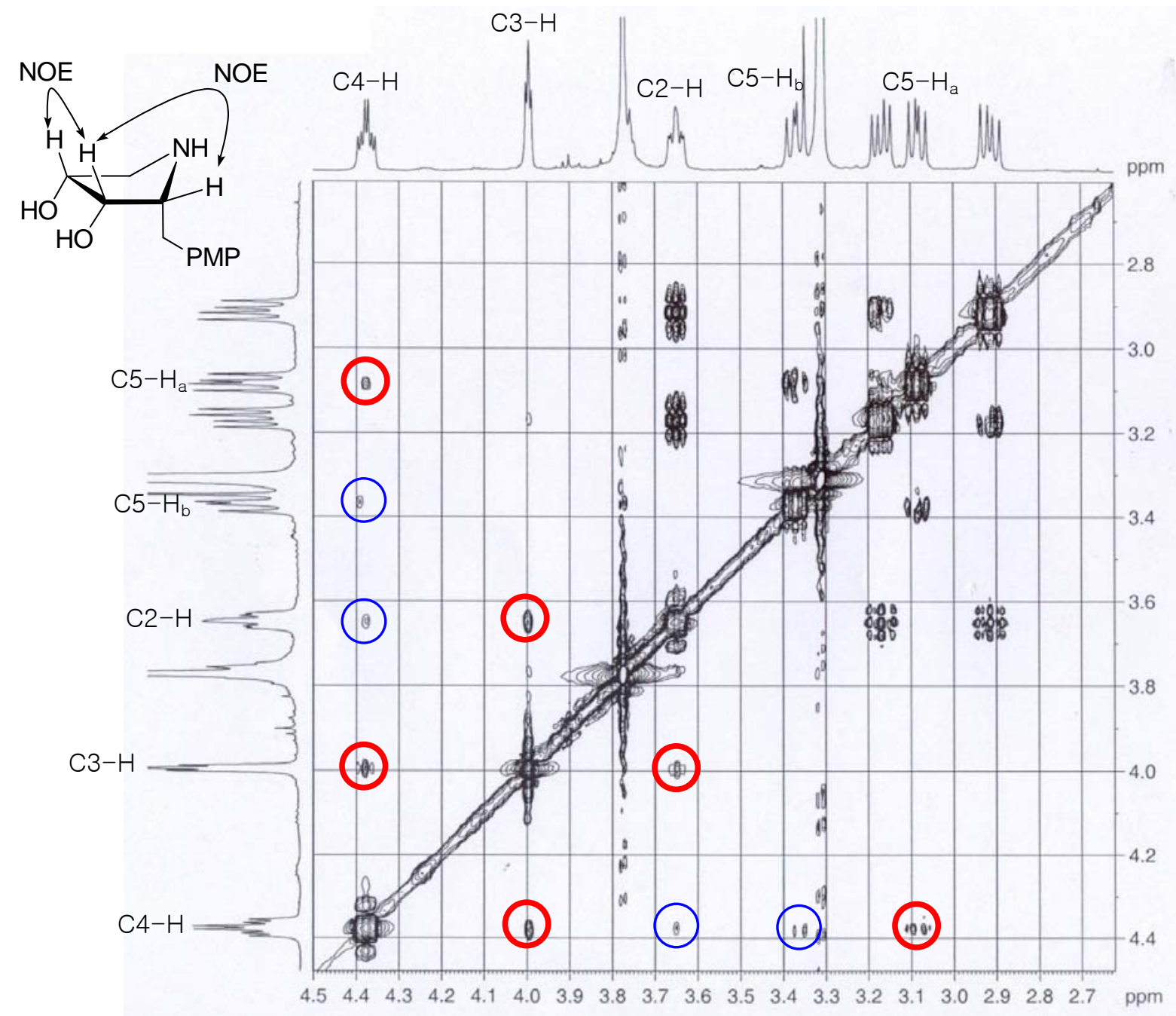

Spectra 43. NOESY of $(2 R, 3 S, 4 R)$-Deacetylanisomycin (5) 


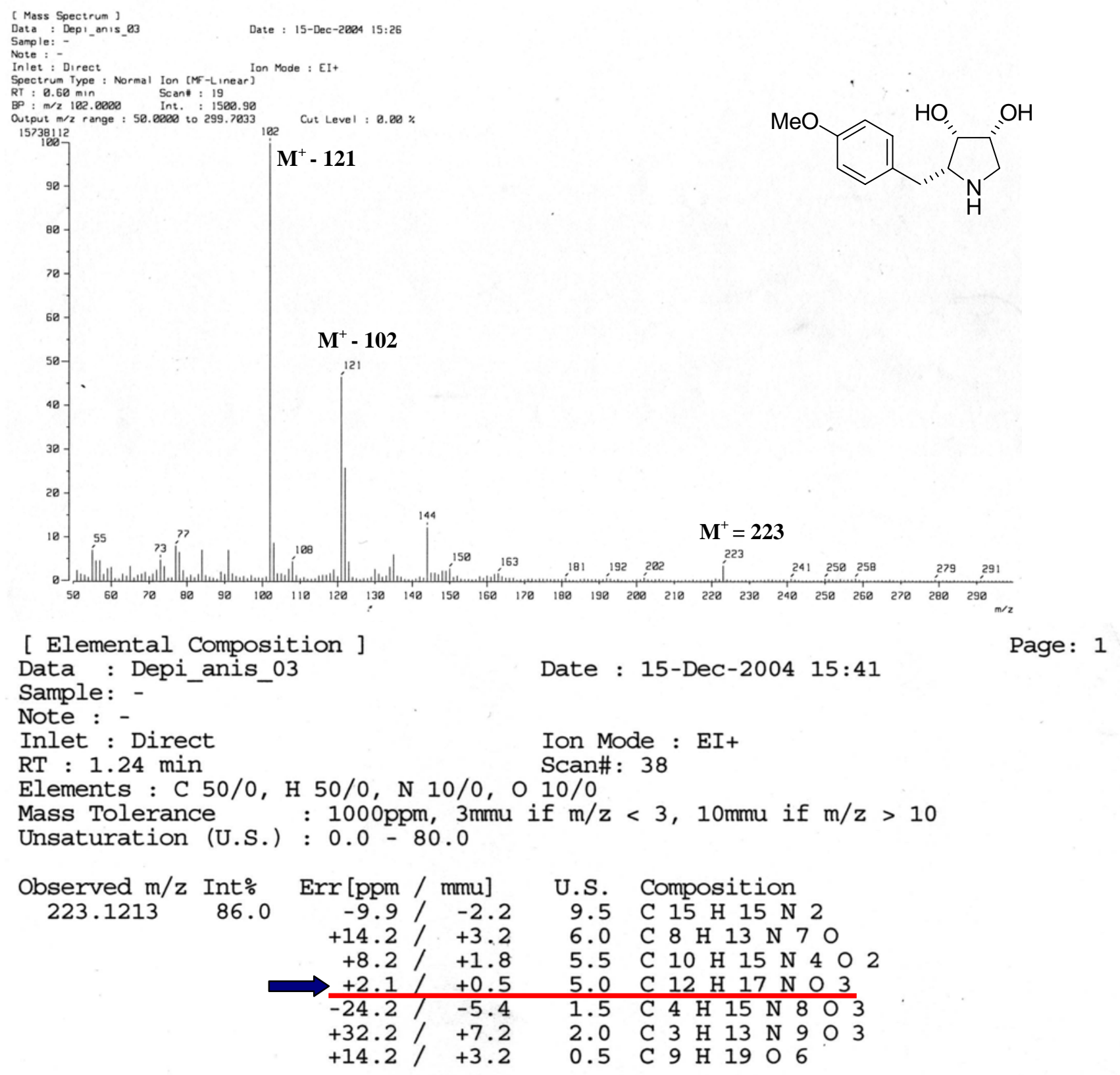

Spectra 44. EI-Mass \& HR-Mass of (2R, 3S, 4R)-Deacetylanisomycin (5) 